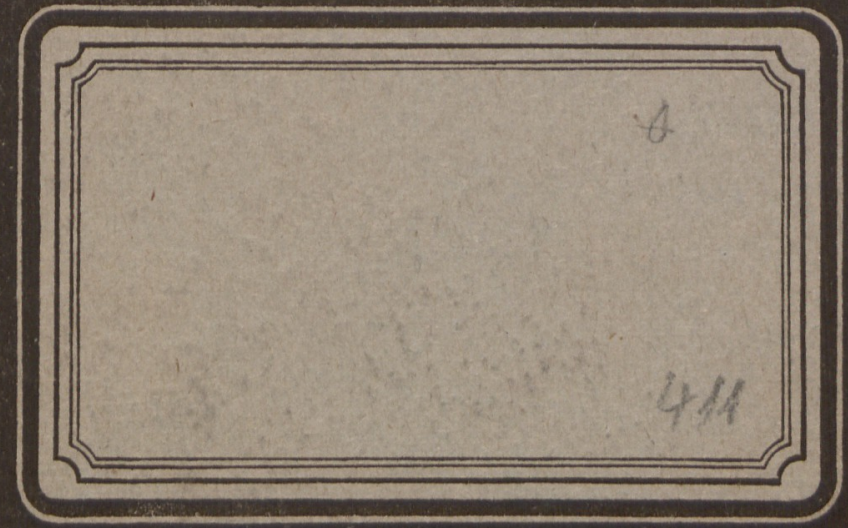




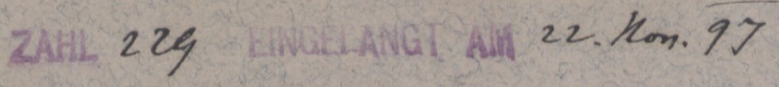

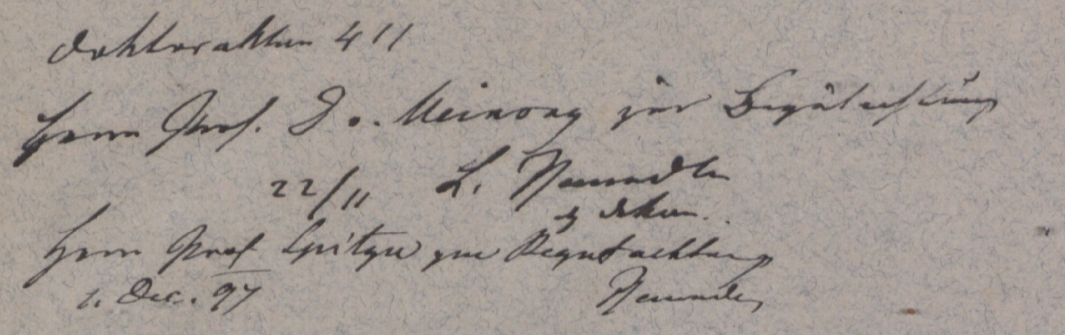

\section{Sonderabdruck}

\section{aus dem \\ Archiv für Geschichte der Philosophie \\ in Gemeinschaft mit}

Hermann Diels, Wilhelm Dilthey, Benno Erdmann und Eduard Zeller

herausgegeben

von

L u d w ig Stein.

B a n d VIII, H e f t 3. 


$$
\frac{\pi}{250,161}
$$

\section{Univoraitate- Elblibiudk Q.र⿰丿乛工}




\section{Die Polemik Alexanders von Aphrodisia gegen die verschiedenen Theorien des Sehens.}

Von

Joh. Zahlfleisch in Ried.

I.

Die im Gegensatze zu dem im 1. Buche vorkommenden positiven Lehren oder Erklärungen im 2. angestellte Polemik gegen die Vorgänger des Aristoteles hat einen für die Geschichte der Physiologie der Sinne und für die Geschichte der Philosophie überhaupt bemerkenswerten Inhalt. Alexanders Polemik scheidet sich in mehrere Abtheilungen.

A. Die Lehre vom Gesichtssinn mit Rücksicht auf die Strahlentheorie.

Aristoteles hat in den Problemen sectio XI num. 58, 905 a 37 der Ansicht Ausdruck gegeben, dass die Fortpflanzung des Lichtes nach Art der Sonnenstrahlen geschieht. Auf diese Anschauung nimmt Alexander (Supplem. 2. Buch 127, 27 ff.) Rücksicht, indem er sich gegen diejenigen erklärt, welche die Behauptung aufstellen, dass das Sehen auf Grund der Aussendung von Strahlen stattfindet. Alexander meint, dass man dies nur dann sagen dürfe, wenn diese Strahlen etwas Körperliches sind. Denn dann frage es sich, ob dieses letztere Luft, Licht oder Feuer sei. Zugleich könne es der Fall sein, dass ein solcher Strahl etwas Zusammenhängendes sei, oder etwas Getrenntes. Das erstere könne dann in dem Sinne vonstatten gehen, dass entweder durchgehends dieser Zusammenhang vorhanden sei, oder so, dass der Strahl bei seinem 
Ausgehen aus der Lichtquelle getrennt sei, dann aber die Theile desselben wieder sich vereinigen.

Gegen diese Voraussetzungen nun gibt Alexander zu bedenken, dass einmal aus Luft ein solcher Strahl nicht bestehen könne, da man denselben dann von der übrigen Luft gar nicht zu unterscheiden vermöchte. Wäre der Strahl ein aus Licht oder durchsichtiger Luft gebildeter Körper, dann liesse sich auch unter dieser Voraussetzung ein Unterschied zwischen dem Lichtstrahl und dem bereits vorhandenen Lichte gar nicht denken. Und weil das Auge die Eigenthümlichkeit besitzt, dass Licht von demselben ausgeht in der Weise, dass es sogar bis zu den Gestirnen dringt, eine Annahme, welche sonst dem Empedokles zugeschrieben wird, so müsste man, wenn diese Strahlen wirklich Licht wären, selbst bei Nacht und in der Dunkelheit sehen, so zwar, dass, wenn diese Lichtstrahlen schwächer wären als das Tageslicht, umsomehr ein Sehen bei Nacht und in der Dunkelheit stattfinden müsste, je weniger leicht dasselbe am Tage zustande kommen könnte, weil das schwächere Licht durch das stärkere absorbirt wird, ungefähr in der Weise, wie dies bei den von uns Modernen mit dem Namen der phosphorescirenden belegten Körpern geschieht. Und wenn schon von einem einzigen Strahl aus ein Sehen nicht stattfindet, dann frägt es sich, weshalb denn nicht mehrere so schwache Strahlen sich vereinigen, um in ähnlicher Weise den Erfolg zu sichern, wie da, wo mehrere Kerzen zusammenwirken, um einen gewissen Lichteffect hervorzubringen. Ausserdem (128, $10 \mathrm{ff}$.) muss man es sonderbar finden, weshalb wir nicht unsere Gesichtsenergien ("̋'ֻsıs) sehen, d. h. die aus den Augen unter der Voraussetzung der wirklichen Empfindung hervorgehenden Lichtstrahlen, da gerade das Gegentheil davon stattfindet, indem wir weder bei Tage noch bei Nacht eine derartige Wahrnehmung machen. Und nun bleibt also noch das Feuer $(128,12 \mathrm{ff}$.). In diesem Falle wäre der Lichtstrahl warm und verbrennlich, wovon aber nichts bekannt ist, also dass man auch nicht von feurigen Ausströmungen zu sprechen braucht, weil es nicht nothwendig ist, dass eine Beleuchtung durch Mitwirkung des Feuers stattfindet. Dazu kommt, dass ein solcher feuriger Lichtstrahl, wenn er auf Wasser fällt, erlöschen müsste, was nicht der 
Fall ist, weil wir ja auch im Wasser sehen; gerade so wie die anderen Thiere. Ferner müsste, wenn die Strahlen feurig sind, vermöge der nach aufwärts dringenden Kraft des Feuers ein Sehen in dieser Richtung leichter vor sich gehen als nach abwärts, wovon aber wieder nichts zu spüren ist. Wenn ferner $(128,20 \mathrm{ff}$.) ein Sehen dadurch vor sich geht, dass die Lichtstrahlen, welche vom Auge zu dem gesehenen Gegenstande gelangen, den letzteren berühren, dann frägt es sich, weshalb da das Sehen dann ein Tasten ist, nicht auch Warmes und Kaltes durch den Gesichtssinn wahrgenommen wird, weil dabei vorausgesetzt werden muss, dass man doch eher die dem Tastsinn unterliegenden Objecte als die Farben, die man nicht mit dem Tastsinn wahrzunehmen vermag, mittelst der so gearteten Strahlen empfindet. (Wer denkt hiebei nicht unwillkürlich an den bekannten Satz, dass das Sehen ein in die Ferne gehendes Tasten sei, wodurch der Einwendung unseres Commentators eine Instanz entgegengehalten würde?)

Alexander geht jetzt auf die zweite Reihe seiner Einwendungen über. Er sagt $(128,24 \mathrm{ff}$.): Wenn der den wirklichen Gesichtseindruck hervorrufende Strahlenkörper ein zusammenhängender wäre, dann müsste der Raum, in welchem die Pupille sich befindet, in seiner ganzen Ausdehnung ein Gang sein, durch den der Lichtstrahl durchdringt, wovon man aber nichts wahrnimmt. Und wenn auch ein zusammenhängender Strahl ausgesondert wird, wie kommt es, dass dieser Lichtstrom sich nicht spaltet und in der äusseren, dünneren Luft (wo also kein Halt mehr für den Strahl gegeben ist) sich zerstreut? Oder wenn eine solche Zerstreuung nicht stattfindet, warum rückt er nicht in eine Enge zusammen (also dass er sein Ziel nicht erreicht), während er immer weiter vorwärts dringen will? Denn das finden wir auch bei den anderen Körpern, wie z. B. Wasser bei seinem Vorrücken entweder immer weiter sich ausbreitet oder in eine Enge sich verliert, sowie die Quellen, obwohl sie aus dichterem Stoffe bestehen als Lichtstrahlen (und infolge dessen leichter sich Bahn brechen sollten); die Flamme aber endigt immer in eine Spitze (also dass man dies auch bei den dem Feuer so ähnlichen Lichtstrahlen voraussetzen sollte). End doch kommt gerade das Gegentheil vor, indem der Lichtstrahl, der vom 
Auge ausgeht, sich kegelförmig immer mehr verbreitert, je weiter er sich vom Auge entfernt (offenbar hat der Commentator dabei vorausgesetzt, dass es mit den in Rede stehenden Lichtstrahlen sich gerade so verhalte, wie mit denjenigen, welche man in der Dunkelheit aus einem Lichte hervorgehen sieht; denn es ist das ein förmliches Strahlenbündel, welches von der Lichtquelle nach aussen in divergierenden Linien hervorgeht), ohne dass man etwas Derartiges weder bei dichten noch bei dünnen Körpern wahrzunehmen im Stande ist.

Ueberhaupt, wenn man voraussetzt, dass die Lichtstrahlen als etwas Körperliches betrachtet werden müssen, dann wäre es nothwendig, dass sie einen Raum einnehmen, während doch dabei die Annahme gemacht werden müsste, dass ein Körper in den anderen eindringt, oder dass ein leerer Raum von dem übrigen abgeschlossen

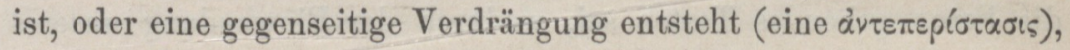
sei es dass Luft oder Wasser von dieser Verdrängung betroffen wird. Es müsste also in dem hinter der Pupille befindlichen Raume Wasser sein, welches verdrängt wird, ohne dass man eine solche Annahme begreiflich findet. Ja auch wenn man den Lichtstrahl mit dem Athem sich vertauschen lassen wollte, so wäre dies zuviel behauptet, wenn man bedenkt, dass man im Wasser, wo selbst auch ein Sehen stattfindet, nicht athmet, und dass überhaupt die Wasserthiere nicht athmen, wenn man einige davon ausnimmt. Und wollte man den ganzen hinter der Pupille befindlichen Raum mit Wasser sich angefüllt denken, an dessen Stelle der Lichtstrahl tritt, dann würden wir nicht mit diesem ganzen Raume diese Aussendung veranstalten, indem wir ja auch nicht mit diesem ganzen Raume sehen, sondern es müsste eine bestimmte Stelle von einer gewissen Ausdehnung vorhanden sein, in welcher diese Vertauschung vor sich geht. Und daraus ergäbe sich der Schluss, dass es nur ein Theil des zum eigentlichen Gesichtsorgane gehörigen Raumes ist, mit welchem wir sehen, während wir mit einem anderen wieder dies nicht zu thun vermögen. Endlich wenn die Lichtstrahlen etwas derartig Körperliches sind, das im Gesichtsorgane sich gebildet hat, dann wäre kein Grund vorhanden, warum die nicht mit einem Augenlide versehenen Thiere im Schlafe nicht 
sehen, da dieselben ja die Gänge des Sehkörpers mit Lichtstoff angefüllt haben müssen. (Hieraus ergibt sich, dass die von Bruns in der kritischen Anmerkung vorgeführte Conjectur $\varepsilon^{\prime} \gamma \varepsilon \mu \eta \dot{\eta} \pi \lambda \eta_{p}^{\prime} \iota \iota \varsigma$ unhaltbar erscheint.)

Es folgt die Behandlung der letzten Annahme, dass nämlich (129, 9 ff.) die Lichtstrahlen nicht zusammenhängend, sondern getrennt sich bilden, dann müsste diese Trennung entweder wieder aufgehoben werden oder so bleiben, wie sie war. Im ersteren Falle müsste man wieder fragen, wie es denn möglich ist, dass ein kegelförmiges Büschel, welches mit seiner Grundfläche sogar den grössten Theil des Himmels bedeckt, sich auf einmal so verenge, dass man von diesem Kegel gar nichts mehr wahrnimmt. Und doch sollte ja das Gegentheil der Fall sein, nämlich dass schon von allem Anfang an eine Zusammenziehung stattfinde, die von der umgebenden Luft eingeleitet wird. Und wenn die Strahlen nicht aus einem einzigen in sich zusammenhängenden und für sich bestehenden Körper gebildet sind, wenn sie vielmehr kegelförmig auseinandertreten, dann müsste in der Mitte des von solchen Strahlen eingefassten Raumes eine dunkle Fläche entstehen, so dass, weil der Strahlenraum nicht vergrössert erscheint (darnach müsste es bei dem $\mu \grave{\eta} 129,16$ bleiben), in der Mitte nichts gesehen wird, ja sogar ein grösserer Raum unsichtbar bleibt als sichtbar; und sollte auch der eingeschlossene Raum gleich gross sein wie der Strahlenraum, dann könnte man immer noch ebensoviel sehen als nicht, während die Annahme eines solchen eingeschlossenen Raumes, der kleiner ist als der bestrahlte, ein Ding der Unmöglichkeit wäre. Und wie sollten so dicke Körper, wie hier die Strahlen (vermöge des durch sie gebildeten Strahlenkegels) vorausgesetzt werden, von einem so kleinen Dinge, wie der Lichtbrechapparat des Auges ist, hervorgehend $(129,20 \mathrm{f}$, damit ist allerdings nur ein speculativer Beweis geliefert, ohne dass man jedoch demselben seine Berechtigung auf Grund allgemeiner Erfahrung absprechen darf)? Und warum bleibt dieser Strahlenkörper immer in gerader Richtung, ohne dass er von Winden oder stark bewegtem Wasser hin- und hergerüttelt wird, wenn nur die Wasserebene sich nicht verändert (Bruns sagt in der Anmerkung dazu: "de rapidis fluviis dicere vi- 
detur, quorum vadum perlucet, dumne superficies perturbata sit. Sed plura exciderunt." Die letzteren 3 Worte hätte sich Bruns ersparen können, weil auch ohne dass noch etwas vermisst wird, der Sinn klar genug ist)? Zudem muss darnach gefragt werden, wie die Strahlen durch ein festes durchsichtiges Mittel sich hindurch begeben. Wenn man nämlich sagen wollte, dass solche feste Mittel Gänge haben, dann handelt es sich darum, zu erkennen, ob dieselben leer sind, da denn dieselben als mitten im Festen eingeschlossene Oeffnungen gedacht werden müssten (was in diesem Falle unmotiviert wäre), oder ob sie mit Luft oder mit einem anderen Körper erfüllt erscheinen, welcher jedoch dann weichen müsste, wenn der Strahlenkörper eindringt; dann frägt es sich aber: wohin damit? Und wenn nun mehrere Körper durch einen und denselben Gang hindurch dringen müssen, dann frägt es sich, wie denn mittelst eines und desselben Ganges mehrere Personen zugleich gesehen werden können (der Gedanke ist: Man muss die Frage erheben, wie denn mehrere Objecte zugleich wahrgenommen werden, da man von jedem derselben einen Strahlenkörper ausgehen lassen müsste, welcher durch denjenigen Gang hindurch sich drängt, welcher für das gleichzeitige Sehen zweier in einer und derselben Gesichtslinie befindlichen Körper prädestiniert ist). In diesem Falle müssten aber zwei Körper gleichzeitig in demselben Raume sich befinden, was nicht angeht. Man könnte dem gegenüber einwenden, dass die beiden Objecte doch nicht in einer und derselben Linie liegen, weil sie sich ja dann gegenseitig verdecken müssten, also dass nur ein einziger Strahlenkörper angenommen werden könnte. Schwerer wiegt aber die Einwendung, welche man von dem modernen physiologischen Standpunkte aus zu erheben vermag, dass ein gleichzeitiges Sehen zweier Objecte überhaupt nicht möglich ist, indem das Auge, wenn auch mit ungemein raschen Bewegungen in der Weise von einem zum anderen Objecte wandert, dass in Wirklichkeit keine Gleichzeitigkeit, sondern ein Nacheinander vorausgesetzt werden muss. Zugleich ergibt sich aus

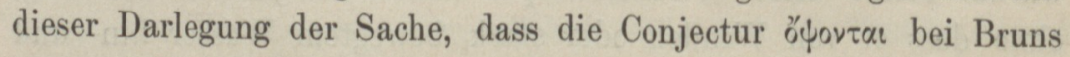
zum mindesten überflüssig ist. Dazu muss bemerkt werden, dass man nicht ersehen kann, wie auf diesem Wege das Durchsichtige 
als zusammenhangend erblickt zu werden vermag. Denn nach der erwähnten Theorie müssten die Poren allein gesehen werden oder die durch die letzteren abgeschlossene Fläche des Durchsichtigen, sowie das bei den Schläuchen der Fall ist (wenn man durchsieht). Und ebenso müsste auch das nur durch Poren gesehene Durchsichtige dann verdunkelt werden, wenn gerade keine solchen Gänge in denselben sich befinden. (Daran hat aber Alexander nicht gedacht, dass diese Poren allenthalben im Durchsichtigen verbreitet sind.) Und wie lässt sich denken, dass ein solcher Körper in uns ist, dass er sogar bis an die Gestirne hinaus gesendet wird, so dass man annehmen müsste, er habe seinen Platz an einer Stelle, die man doch wohl nicht auffinden kann, weil man nicht annehmen darf, derselbe sei schon da, bevor wir die Gesichtsempfindung haben (hätte Alexander eine Ahnung von dem Lichtäther gehabt, dann wäre ihm die Vorstellung dieser Thatsache klarer geworden)? Und wie ist es möglich, dass man sozusagen urplötzlich und zugleich mit dem Sehen jenen Strahlenkörper aussendet (auch diese Eigenthümlichkeit hätte Alexander aus dem eben hervorgehobenen Lichtäther verstehen lernen können)? Und wie könnten sich, meint Alexander, zwei Personen, die sich zufällig gegenüber treten, einander sehen, da die Strahlenkörper sich gegenseitig verdecken, oder in einander eindringen oder nur der eine von beiden sichtbar wird, weil er von dem anderen überwältigt wird? Wenn endlich die Thatsache zum Vorschein kommt, dass die Strahlenkörper, die in der angegebenen Weise nothwendig erscheinen, nur durch eine Bewegung möglich sind, dann ist es auffallend, weshalb das Sehen der Gegenstände ohne Rücksicht auf ihre Entfernung vom sehenden Subject gleichzeitig vonstatten geht, da man doch annehmen sollte, dass jene Bewegung bis zu den Gestirnen länger dauert als die in die nächste Umgebung gerichtete. -

Ueberblicken wir die ganze Polemik gegen die in Rede stehende Theorie, so finden wir das Eine, dass dem Alexander weder die moderne Lehre von dem Lichtäther noch jene von der Geschwindigkeit des Lichtes bekannt war. Denn namentlich der letztere Umstand hätte ihm nicht bloss die Unhaltbarkeit der ganzen Strahlentheorie, sondern auch die Unrichtigkeit der zuletzt von ihm 
vorausgesetzten Prämisse gezeigt, dass man nähere und entfernte Gegenstände gleichzeitig in den Blickpunkt bekommen kann. Trotzdem darf man nicht so obenhin über diese nur als Verirrung vorkommende Theorie der Alten den Stab brechen, weil in der That auch die moderne Optik eine wirkliche Materie, den Lichtäther, zur Grundlage aller Empfindung und Fortpflanzung des Lichts gemacht hat. Verhält es sich ja mit den Fortschritten auf diesem Gebiete genau so, wie mit anderen, z. B. in der Astronomie, wo man zwar die eigenthümlichen Hilfsmittel heutzutage belächelt, die von den Alten angewendet wurden zu dem Zwecke, die verwickelt scheinenden Bewegungen der Gestirne zu erklären, ohne dass man in Abrede stellen darf, dass ein allmählicher Fortschritt $\mathrm{zu}$ den heutigen Errungenschaften auf andere Weise als auf dem Wege annähernder Vermutungen und Combinationen, also durch verschiedene Irrungen nicht möglich war. Vgl. Gruppe, die kosmischen Systeme der Griechen. Berlin 1851 S. 124. 216. $217 \mathrm{f}$.

\section{B. Die Lehre von der Verbreitung des Lichtes durch An- spannung der Luft.}

Diese Theorie besteht darin, dass die auf den Lichtbrechapparat aufsitzende Luft durch die lebendige Kraft des Gesichtssinnes

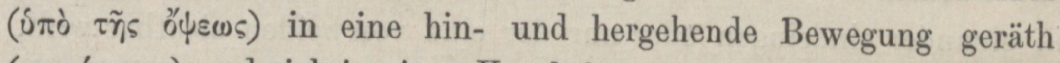

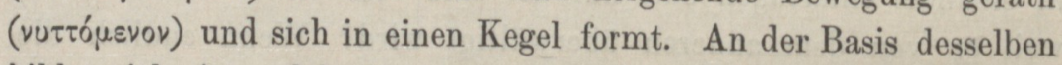
bildet sich eine solche Formation desselben, dass er genau diejenige Eigenthümlichkeit zeigt, welche in dem Objecte des Sehens vorhanden ist, also dass auf diese Weise der Gesichtseindruck in genau adäquater Art entsteht, ähnlich wie man mittelst des Stockes und Tastsinns das Oberflächenbild der Unebenheiten auf dem Erdboden u. dgl. wahrnimmt. Diese so sehr an unsere modernen Anschauungen anklingende Theorie wird von Alexander (130, 18 ff.) zurückgewiesen, indem er sich vor allem dahin ausspricht, dass man bei Annahme derselben sowohl den Zug vom Sinnesorgan als auch den vom Object aus wahrnehmen müsse, obschon dies wegen der Dünnheit der Luft nicht möglich sei. Zugleich ergebe sich ein Widerspruch dadurch, dass wir auch dann sehen, wenn wir einige Schritte zurücktreten, während dies doch dann unmöglich sei, wenn ein be- 
stimmtes Aufsitzen des gesehenen Bildes auf dem Sinnesorgan vorausgesetzt werden muss, so dass wir nur in einer bestimmten Entfernung das Bild vom Objecte haben können (wer erinnert sich in diesem Falle nicht an die Theorie von der Accommodation des Auges, welche ja eben zu dem Zwecke vorhanden ist, dass wir auf verschiedene Entfernungen hin zu sehen vermögen?). Und wenn man den Ausgang von dem gesehenen Object nimmt, wäre anzunehmen, dass, wenn nicht die bestimmte Entfernung eingehalten wird, ein Abstand des zuletzt möglich gewordenen Bildes vom Auge vorhanden ist, der hier ebensowenig am Platze erscheint, wie wenn man aus grösserer Entfernung eine bestimmte Tastempfindung haben sollte? Und dann wäre es inconsequent, nur beim Gesichtssinne eine solche Wirksamkeit des $\pi v \varepsilon \tilde{u} \mu \alpha$, jener Luft, anzunehmen, in welcher die Fortpflanzung des erwähnten Gesichtsbildes vor sich geht, indem ja auch für andere Sinne dieselbe Art der Empfindung durch Fortpflanzung angenommen werden müsste. (Man denkt hier vor allem an den hypothetischen Lichtäther, welcher unter dem Begriffe jenes $\pi \nu \varepsilon \tilde{\mu} \mu \alpha$ verstanden werden kann; zugleich darf aber hier jener Entgegnung bei Galen de plac. Hipp. et Plat. 642, 12 (Iw. Müller) gedacht werden, wo der Analogie mit dem Tastsinn insofern entgegengetreten wird, als man durch das Auge zugleich von der Farbe, der Grösse und Umgebung des Objects Kunde erhält, was beim Tastsinn nicht möglich sei. Wir Modernen wissen, dass dies nicht ganz richtig sich verhält. Und wenn auch bei Aristoteles die Grösse, Bewegung u. dgl. durch den sogenannten Gemeinsinn empfunden werden, so sind das doch nur secundäre Auffassungen der Psyche, während die Farbenempfindung im wahren Sinn des Wortes vermöge der Schwingungstheorie nach denselben Kriterien beurtheilt werden muss, wie die Empfindung des Lichtes, d. h. die Gesichtsempfindung überhaupt. Und gesetzt den Fall, man erkläre sich die letztere durch eine Art Tastempfindung auf Grund des in verschiedenen Schwingungen zum Auge gelangenden Lichtäthers, so ist darin die Farbenempfindung eo ipso eingeschlossen.)

Alexander wendet aber weiterhin ein, dass man diese Theorie deshalb nicht gelten lassen könne, weil dann ein Gleichartiges sich selbst bewegen müsse, was auf keine Art von Bewegung passe 
(mit dieser Behauptung meint Alexander doch wohl nichts weiter, als dass die Luftschwingungen sich nicht fortpflanzen können, ausser es bewege sich die Luft durch sich selbst, also ohne einen ausserhalb ihrer befindlichen Anstoss. Vgl. dazu Aristoteles Physik $(\Theta) 5$ mit meiner Bemerkung im 100. Bande der Zeitschrift für Philos. und philos. Kritik S.196). Ausserdem, bemerkt Alexander (131, 8ff.), indem er auf die das Weltall bewegenden Kräfte Rücksicht nimmt, es sei undenkbar, dass man die in Rede stehende Theorie für die Gesichtsempfindung aufrecht erhalte, wenn man bedenkt, dass in diesem Falle entgegengesetzt wirkende Kräfte angenommen werden müssten. Denn wenn das hypothetische $\pi \nu \varepsilon \tilde{u} \mu \alpha$ vermöge der im Weltall überhaupt wirkenden Bewegungskraft nach aufwärts getrieben wird, während mit Rücksicht auf den speciellen Fall der von einem oberhalb befindlichen Objecte auf das unterhalb desselben gelegene Auge ausgehenden Bewegung jenes $\pi \nu \varepsilon \tilde{\mu} \mu \alpha$ abwärts gehen sollte, so müssen sich beide Bewegungen gegenseitig mindern oder gar aufheben. (Natürlich beruht diese Polemik nur auf der Voraussetzung einer Bewegung des Weltalls, wie man sie bis heute noch nicht hat entdecken können.) Hierauf folgt (131,22ff.) der bereits oben von Galen gemachte Einwand, der eigentlich gegen die Stoiker gerichtet ist, so dass man annehmen muss, dass alle die hier vorgebrachten und zurückgewiesenen Lehren aus stoischen Quellen stammen. Wenn aber Alexander 131, $28 \mathrm{f}$. den Schluss zieht, dass unter Voraussetzung der von ihm widerlegten Lehre das Sehen nichts anderes als ein Tasten wäre, dann hat er vollkommen recht; man könnte dagegen nur sagen, dass trotzdem die neuere Psychologie das Sehen als ein Tasten in die Ferne bezeichnete, obschon nur von ferne die in diesen Worten liegende Analogie sich bewahrheitet, wie sie denn auch von den maassgebenden Persönlichkeiten auf diesem Gebiete zurückgewiesen wurde.

Die folgende Frage lautet, wie es denn trotz der hier vorausgesetzten Theorie möglich werde, dass man aus dem Dunkel wohl solche Gegenstände erkenne und sehe, welche im Lichte sich befinden, dagegen nicht umgekehrt von einer beleuchteten Stelle aus in Dunkelheit gehüllte Dinge wahrzunehmen vermöge. Der gewöhnlich für diese Erscheinung vorgeführte Grund, dass vermöge der genauen 
Unterscheidung der Sehobjecte in der beleuchteten Luft eine grössere Kraftthätigkeit für den Zweck des Sehens erzeugt wird, während die lichtleere Luft den Gestalten der Objecte keinen Halt verschaffen kann (wenn man auf solche Weise das $x \varepsilon \chi \alpha \lambda \tilde{\alpha} \sigma 0$ a, , 131, 34. 132, 1. $13 \mathrm{f}$. erklärt, bedarf es keineswegs der von Bruns nothwendig erachteten Hinzufügung eines $\left.\mu \eta_{i}\right)$, ist nichtig. Denn man muss bedenken, dass, wenn auch die nicht von Licht durchzogene Luft, wie jene Annahme lautet, als eine dichtere bezeichnet wird, doch auf solche Weise eher jener zum deutlichen Sehen durch die Luft nothwendige Widerstand erzeugt wird, zumal wenn man gerade deshalb, weil das Auge im Dunkeln sich befindet, beleuchtete Gegenstände (offenbar wegen des auf jenem Widerstande beruhenden grösseren Contrastes) deutlicher und besser wahrzunehmen im Stande ist. (Das hier zu Grunde gelegte Phänomen verdient gewiss eine genauere Untersuchung; doch dürfte diese Thatsache, dass man aus dem Dunkel heraus besser sieht als dann, wenn das Auge gleichfalls beleuchtet wird, vorzugsweise auf schwach beleuchtete Gegenstände passen, weil in solchem Falle natürlich der gegenseitige Wettstreit des Lichtes im Objecte und desjenigen, in welchem der Beschauer sich befindet, wegfällt und nur das erstere beachtet wird.) Und zur näheren Ausführung des so eben vorgeführten Gedankens wird von Alexander (131, 37 ff.) die Ansicht aufgestellt, dass es ein Widerspruch sei, anzunehmen, dass nur derjenige Luft-Lichtstrahl eine Wirkung im Auge erzielt, welcher unter dem Einfluss des Lichtes sich befindet, und daneben zu sagen, dass die lichtleere Luft keinen Halt für das Gesichtsbild gewährt. Denn dann sei es gleichgiltig, ob man diese haltlose Strecke am Object oder am Auge voraussetzt. Denn es sei selbstverständlich, dass auch dann, wenn die am Lichtbrechapparat befindliche Lichtluft dunkel, die aber am Objecte hell ist, die Sehkraft, welche von dem Gesichtsorgane ausgeht, erlahmt, da sie zunächst durch die schwer durchdringliche unbeleuchtete Schicht sich Bahn brechen möchte. (Solche Anschauungen sind nun aber nur dann erklärlich, wenn schon von vornherein ein Unterschied zwischen Beleuchtungsstoff und derjenigen Luft angenommen wird, in welcher dieser Stoff fehlt. Wir Modernen wissen, dass der „Beleuchtungsstoff“ überall im Weltraum 
als Lichtäther verbreitet ist, daher auch in der Luft sich befindet.) Natürlich fällt (mit Rücksicht auf die so eben in der Parenthese von mir gegebenen Erklärung) auch der folgende Einwand, dass die Bewohner zweier gegenüber befindlichen nur durch einen dunklen Raum in der Mitte von einander getrennten Häuser, in welchen Licht zum Vorschein kommt, dieselben sehen, obwohl dieser dunkle Raum nach der behandelten Theorie eigentlich die Durchdringung des Lichtes verhindern sollte.

Die Ursache ferner, warum wir die Gestirne des Nachthimmels am Tage nicht sehen, was doch nach dem eben von Alexander Erwähnten, wenn die Theorie der Gegner richtig wäre, selbstverständlich sein müsste, weil nur in der Nacht das jedes Sehen hindernde Dunkel vorhanden ist, spricht ebenfalls nach Alexander 132, 10 ff. gegen die erwähnte Lufttheorie. Denn gerade durch die grössere Festigkeit des Dunkels und das derbere Gefüge der erwähnten Nachtluft sei man im Stande die Gestirne zu sehen, was bei der in ihren inneren Theilchen verschiedene Objectsdifferenzen leichter zum Vorschein kommen lassenden und daher nach der Meinung der Gegner mit energischerer Kraft wirkenden Tagluft nicht möglich werde, weil wir mit derselben keinen richtigen Haltpunkt gewinnen und dadurch nicht im Stande sind, der Fähigkeit unseres Gesichtssinnes diejenige Thatkraft zu verleihen, welche ihn in den Stand setzt, auf so weite Entfernungen hin seine Wirksamkeit auszuüben. Und das sei eben aus den erwähnten Ursachen bei Gelegenheit von Sonnenfinsternissen oder in einem schattigen Walde sowie in tiefen Brunnen der Fall, da wir dann trotz der Tageshelle die Gestirne sehen (die Gründe für die zuletzt aufgeführten Phänomene liegen natürlich ganz anderswo als in dem von Alexander gemeinten Umstande).

Hören wir aber weiter: „Wenn wir die im Wasser befindlichen Gegenstände sehen, dann frägt es sich, ob das Wasser im Verein mit der Luft die Sichtbarkeit befördert oder nicht. Denn wenn das letztere der Fall ist, dann kann man diese Theorie des Sehens, weil wir ja auch die Dinge im Wasser wahrnehmen, deshalb nicht anerkennen, weil man nicht verstehen könnte, wie in der Luft ein Entgegenstemmen der Sehkraft (nach 130, 14 ff.) möglich, ja noth- 
wendig ist, während dies im Wasser, wo doch das Sehen jener Gegenstände stattfindet, nicht mehr angenommen wird. Wenn aber das erstere vorkommt, dann müsste man, da die Luft doch weniger dicht als Wasser ist, die Sichtbarmachung der Dinge auch in der dunkeln Luft voraussetzen (was eben nach der von Alexander bekämpften Ansicht, wie wir sahen, nicht stattfindet, wenn das Object im Dunkeln und das Auge im Hellen gelegen ist).

Nimmt man jedoch an, dass dies bei der dunkeln Luft deshalb nicht geschieht, weil sie dünn ist, nämlich dünner als Wasser, so müsste dies mit noch grösserem Rechte bei der noch viel dünneren beleuchteten Luft stattfinden“. (Die grössere Dichtigkeit des Wassers ist es aber nicht, wårum wir die Gegenstände im Wasser sehen oder nicht sehen; sondern nur seine Durchsichtigkeit.)

Da wir dann, wenn wir uns im Wasser selbst befinden, manchmal sogar die Dinge auf dem Grunde sehen, so erfolgt auch hieraus, da ja keine Luft zum Auge dringen kann, die Nothwendigkeit des Schlusses, dass wir uns mit der vorausgesetzten Annahme nicht befreunden können, welche darin besteht, dass wir durch Anspannung der mitten zwischen Subject und Object befindlichen Luft zum Sehen gelangen (auch hier gilt natürlich das so eben in der Parenthese Erwähnte). Die Wasserthiere ferner könnten, falls die aufgestellte Theorie richtig ist, nur dann sehen, wenn im Wasser auch Luft sich befindet, die etwa in ihnen selbst enthalten sein kënnte, wenn nicht die Thatsache dagegen spräche, dass die Luft jedenfalls über das Wasser hinauf getrieben werden müsste, da sie immer das Bestreben hat, aufwärts zu steigen (womit dann eigentlich dieser Fall auf den unmittelbar vorhergehenden zurückgeführt ist). Die Annahme, welche von den Gegnern des Alexander gemacht wird, dass ein Sehen nur durch eine Art Anstemmung geschieht, welche in der Luft stattfindet, führt ihn zu folgendem Schluss (132, 30-33): Wenn die Anstemmung angenommen werden muss, dann kann dieselbe nur mit der Annahme des dem Sehen der Objecte zu Grunde liegenden Mittels als eines. Körpers verbunden

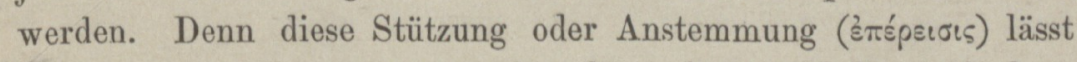
sich nur unter Voraussetzung eines körperhaften Mediums denken. Sofort, wenn man das Licht als etwas Körperloses auffasst, schwindet $26^{*}$ 
386 Joh. Zahlfleisch, Die Polemik Alexanders von Aphrodisia etc.

auch die Möglichkeit jener śmśpsı๘є, die man sich auch da nicht zum Grundsatze machen könnte, wo ein Körper den andern durchdringen, also vernichten müsste, da die Luft ins Wasser einzudringen genöthigt wäre, was deshalb nicht möglich ist, weil zu gleicher Zeit zwei Körper in einem und demselben Raume sich befänden. (Ich glaube wenigstens, dass man sich auf solche Weise das von Alexander Vorgeführte zu ergänzen hat, wenn in den Punkt Beweiskraft gelegt werden soll.) Und wenn das Licht bei der Verdeutlichung, also Discernirung (ótáxpıoıs) der einzelnen Theile des Objects zustande kommt, so sollte man glauben, dass im Falle des

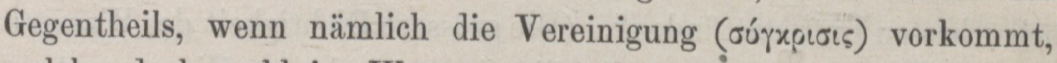
welche doch wohl im Wasser stattfindet, vermöge der Kälte des letzteren das Licht dem Dunkel weicht (diese mit der Lehre, dass das Licht Feuer sei, innig zusammenhängende These muss natürlich, weil eben letzteres nicht der Fall ist, zurückgewiesen werden).

(Sonderabdruck aus dem Archiv für Geschichte der Philosophie

VIII. Band 3. Heft. 1895.)

Druck von Georg Reimer in Berlin. 


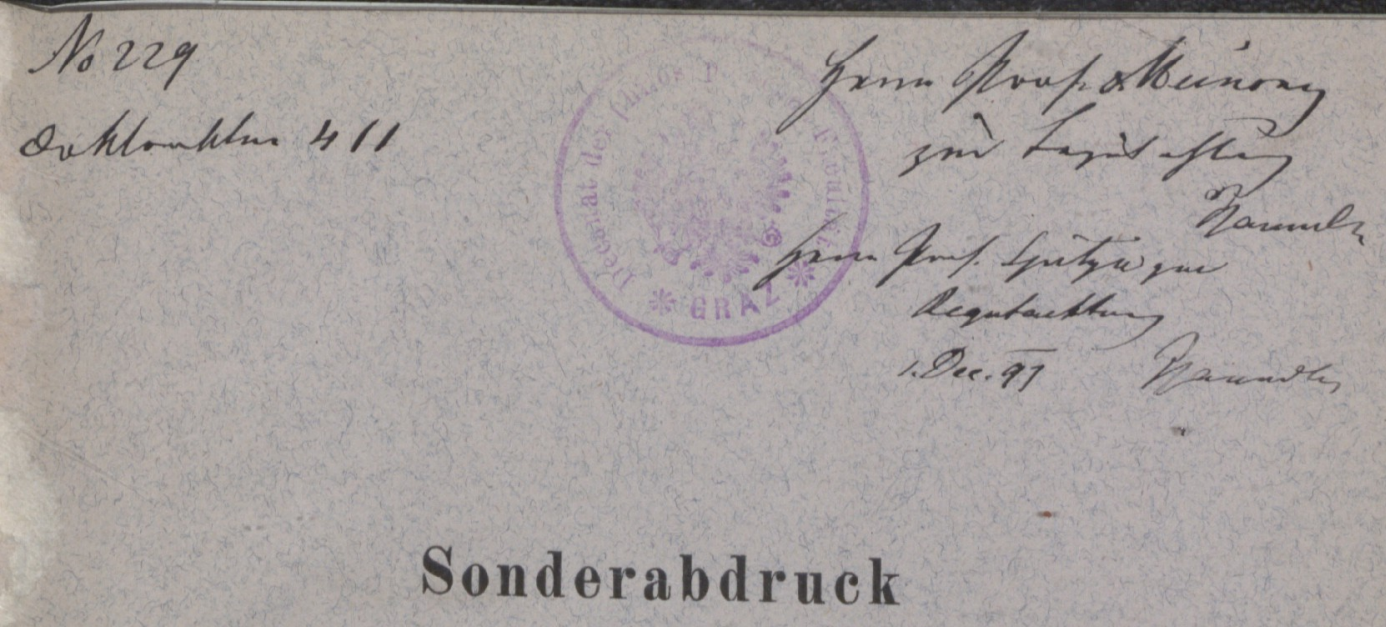

aus dem

Archiv für Geschichte der Philosophie

in Gemeinschaft mit

Hermann Diels, Wilhelm Dilthey, Benno Erdmann und Eduard Zeller

herausgegeben

von

Ludwig Stein.

B a n d VIII, H e f t 4. 


\title{
Die Polemik Alexanders von Aphrodisia gegen die verschiedenen Theorien des Sehens.
}

\author{
Von
}

Joh. Zahlfleisch in Ried.

II.

Alexander polemisirt ferner p. 132, 30-33 gegen die Ansicht derjenigen, welche die auf Grund der änssseren Objectscontouren in der Luft aufgenommene Gestaltung derselben sich bis zum Auge fortpflanzen lassen, dadurch, dass er zu bedenken gibt, dass auf der einen Seite von denselben vorausgesetzt wird, es sei die auf solche Weise heraus kommende Gestaltung etwas derart Körperliches, dass man dasselbe mit der andererseits von ihnen geleugneten Körperhaftigkeit der Luft schwer in Einklang zu bringen vermag. (Und mit Recht. Denn nach den neueren Ergebnissen der Naturwissenschaft hat man nicht zu sagen gewusst, ob der sogenannte Lichtäther etwas Körperliches ist oder nicht, obgleich die allerneuesten Untersuchungen mehr zum Ersteren hinneigen.)

Das Argument p. 132, 33-35 besteht dagegen in einer Zurückweisung der Ansicht, als ob die luftartigen Lichtstrahlen ohne weiteres durch Wasser durchzudringen vermöchten, da doch feststeht, dass nur durch die genaue Unterscheidung der einzelnen bereits geformten Theilchen der Luft das deutliche und genaue Sehen möglich wird, ein Sehen, welches natürlich sofort nicht mehr statthaben könnte, wenn die erwähnten Formen der Theile des Gesichts- 
bildes durch das Wasser zusammengedrückt und unkenntlich ge-

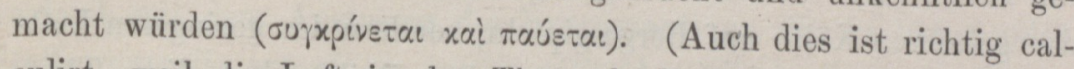
culirt, weil die Luft in der That als Medium des Sehens nicht angenommen werden darf wegen ihrer eigenartigen leichten Verschiebbarkeit der Theile; man sieht das Bestreben, ein Medium des Sehens ausfindig zu machen, ohne dass das gewünschte Ziel zum Vorschein kommt; Alexander hält sich daher wohlweislich in der Defensive.)

Nimmt man ferner an, dass, wenn Wasser gefriert, indem es zu Eis sich verdichtete, eine Arbeit vollzogen wird, welche offenbar schwerer zu vollführen ist, als wenn man einen weniger dichten Körper, wie Luft, voraussetzt, der ja doch eher eine Einwirkung erfahren muss als ein weniger leicht $\mathrm{zu}$ behandelnder, weil dichterer Körper, wie das Wasser im Gegensatz zur Luft einer ist (p. 132, 38 heisst eben die Luft im Gegensatz zum Wasser вù $\pi$ -

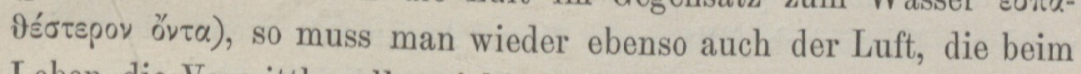
Leben die Vermittlerrolle spielt, die Eigenthümlichkeit zuschreiben, dass sie vermöge der Einwirkung von Kälte nicht mehr brauchbar ist dazu, den Theilchen des Objects zum deutlichen Sehen zu verhelfen, weil ja dann ähnlich, wie beim Gefrieren des Wassers, die Elasticität dahin ist, welche den Lufttheilchen die Form des Objects zuzuertheilen vermag, weil nach dem Gefrieren eine starre Masse allein übrig bleibt. Und wenn wir sehen, dass sogar der dünnste Körper, das Feuer, durch die Eiskälte einen Eintrag erleidet, so ist nicht einzusehen, weshalb dies nicht auch bei der Luft geschehen soll. (Was den letzteren Umstand betrifft, dass Feuer vermöge der Kälte in seiner Wirksamkeit Einbusse erfährt, so hat Alexander vergessen, hierfür auch, wenn gerade nicht die Begründung, so doch die nöthigen Beobachtungen als Beweis anzuführen. Nach gewöhnlicher Anschauung ist nun aber kein Fall bekannt, in welchem Feuer weniger in kalter als in warmer Luft brennen sollte, ausser es kommt-der Feuchtigkeitsgrad in Betracht, welcher wegen früher nicht beachteter, aber aus Grund von Schmelzung anhaftender Eisbildungen beim Holze anzunehmen ist. Der einzige, vielleicht in Betracht zu ziehende Umstand ist der, dass auf hoch gelegenen Orten der Siedepunkt des Wassers ein niedrigerer ist als 
im Thale. Der Grund davon liegt aber nicht in dem Umstande der grösseren Kälte auf Bergesgipfeln, sondern im geringeren Luftdruck, abgesehen davon, dass den alten Philosophen diese Thatsache nicht so leicht bekannt sein konnte. Jedenfalls ist dieses Argument des Alexander nicht stichhaltig. Abgesehen davon darf man die Elemente nicht in jeder beliebigen Hinsicht in eine Reihe stellen, da ja auch bis in die neueste Zeit das Wasser im diametralen Gegensatze zur Luft jedem Versuche seines Zusammendrückens spottete.) Ausserdem bemerkt Alexander 133, 2-4, dass die von ihm angenommene Einflussnahme der Kälte auf Luft und auf das derselben verwandte, dem Sehen zu Grunde liegende $\pi \nu \varepsilon \tilde{u} \mu \alpha$ sich nicht mit der von seinen Gegnern vorausgesetzten Theorie vereinigen lasse, nach welcher die Ursache des Weissen im Schnee jenes in demselben eingeschlossene lichtartige $\pi \nu \varepsilon \tilde{\mu} \mu \alpha$ sei; denn auch dieses müsste ja unter dem Einfluss der Schneekälte zunichte werden. (Natürlich fällt auch dieses Argument, wenn die vorige Annahme Alexanders nicht allgemeine Giltigkeit hat.)

Wenn man ferner die Thatsache erwägt, dass ein solch luftartiges Gebilde, wie das Gesichtsbild nach der Anschauung jener Philosophen ist, auf den geringsten Anstoss hin in Nichts zerfallen muss, so wäre $(133,4-8)$ absolut nicht zu ersehen, dass, wenn einmal dieses irgendwo einen Anstoss erleidet, wie bei der Reflexion des Lichtes im Spiegel oder in den durchsichtigen Glaskörpern

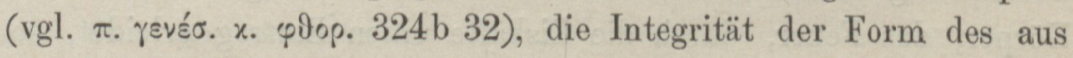
den Lufttheilchen bestehenden Complexes gewahrt bleiben sollte. Kommt das ja auch nicht in dem Bereiche des Tastsinns da vor, wo wir die Gestalt der zu betastenden Oberfläche aus der Ferne mit dem Stocke untersuchen. Denn (das ist wohl der Sinn dieser Stelle) in diesem Falle kommen uns die Objectstheilchen rücksichtlich ihrer äusseren Oberfläche nicht in der Weise zum Bewusstsein, dass wir von denselben ein Gesammtbild bekommen, sondern sofort, wenn wir einen Ort, einen Punkt verlassen haben, tritt an die Stelle dieses vergangenen Eindrucks ein neuer, zweiter, dritter, also dass wir uns den Gesammteindruck nur in der Reproduction sammelnd herstellen, während eigentlich das sinnliche Verbleiben des Tastreizes als solchen in unserer Empfindung als Ergebnis der 
hierbei geltenden Voraussetzung angenommen werden sollte. Denn zugleich mit dem Anstossen des Instruments an die zu betastende Stelle muss die aus einem gewissen Fluid gebildete Form des Tastreizes zunichte werden, weil dieselbe jenem Anstoss eben gar nicht widerstehen kann. Denn sogut wie nicht fest gegründete, weil unterhöhlte oder überhaupt fehlerhaft beschaffene Körper wegen Mangels jeglichen sicheren Stützpunktes sofort zerfallen, wenn ihnen die energische Entgegenwirkung gegenübertritt, welche in einer auf sie Einfluss habenden Macht besteht, geradeso müssen auch die so biegsamen Luftgebilde in ein Nichts sich auflösen, wenn wegen Vorhandenseins einer hindernden Gewalt die Einheit in der Luftformation nicht mehr hergehalten werden kann. - (Und Alexander hat damit auch vollkommen Recht.)

Wenn man ferner annimmt, dass in einem Brunnen (133, 8-14), dessen Wasser durch seine Tiefe verdunkelt erscheint, nur das Bild desjenigen zurückgestrahlt wird, der in den Brunnen hinab sieht, ohne dass er imstande ist, das Wasser selbst zu sehen, so entstünde bei Festhaltung der von Alexander bekämpften Theorie unter gleichzeitiger Voraussetzung der Schwächung des Bildes im reflectirten Lichte ein Widerspruch. Denn man könne nicht ein-

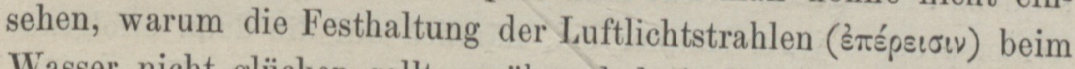
Wasser nicht glücken sollte, während doch die nach der Brechung nicht mehr festgehaltenen Strahlen nach derselben demjenigen sichtbar, weil festgehalten werden, welcher die Brechung erleidet. Es sei dies dasselbe Verhältnis, wie wenn man einen Stab zerbrechen und in zwei Theile spalten wollte, so dass der nicht berührte Theil (Alexander denkt sich, wie Bruns ganz richtig - entsprechend dem für das Wasser im Brunnen gemachten Vergleich - in der Anm. z. St. hervorhebt, dass die Zerbrechung des Stabes so vor sich geht, dass man von ihm die Hälfte entfernt, so dass der Stab bis zur Mitte gespalten erscheint, während die andere Hälfte ganz bleibt und nur die erstere vom Ganzen losgerissen wird [in baculi termino per fracturam orto - in altero eius termino, i. e. in eo, qui ante fracturam proprius erat baculi terminus] doch so, dass man immer noch die Elasticität der Ruthe bei der Berührung ihres Endes fühlen kann) keinen wahrnehmbaren Widerstand mehr leistet, 
während dies bei dem durch die Brechung geschwächten wohl der Fall ist. (Dagegen ist zu bemerken, dass man das Wasser wegen seiner gleichmässigen Oberfläche im ersten Moment nicht sieht, und dass die Spiegelung des Gesichtes nur dieserhalb erfolgt, weil dasselbe von dem äusseren Lichte beleuchtet erscheint, während. das Wasser des Brunnens im Dunkel bleibt.)

Ferner bemerkt Alexander 133, 14-28, dass jene Theorie deshalb nicht stattfinden könne, weil man die Eigenschaft des zum Sehen nothwendigen $\pi v \varepsilon \tilde{\mu} \alpha$, dass es überall durchdringe, gar nicht mit der Thatsache in Einklang bringen könne, dass man nicht im Stande sei durch die Mauern zu sehen. Sagen, dass dies wegen der Festigkeit des Stoffes nicht möglich sei, ist deshalb unzulässig, weil es feste Stoffe, wie Glas, Horn und durchsichtige Steine, gibt, welche das Durchsehen ganz gut gestatten. Will man sich dahin ausreden, dass diese Durchsichtigkeit auf Grund der leichten Zerbrechlichkeit stattfinde, indem solche Körper nicht zu den festen gerechnet werden dürfen, so gilt als Instanz dagegen die Annahme der in solchem Falle nothwendigen Durchsichtigkeit auch von Lehm, der doch ebenso leicht zerbrechlich ist, ohne dass an ihm jene Consequenz Platz griffe. Geht man nun auf die Gegenseite des obigen Widerspruches jener Männer ein, nämlich auf die dann erfolgende Nothwendigkeit, dass die Sehstrahlen nicht auf feste Körper aufschlagen dürfen, wenn man etwas sehen will, so steht dem wieder entgegen, dass der andere Theil jener Theorie sich unmöglich damit vereinbaren lässt, wonach das Bild durch Aufprägung der einzelnen Eindrücke ein deutliches werden soll. Denn dünne und flüssige Körper, deren Theile leicht verschiebbar sind, können unmöglich diese Eindrücke festhalten. (Mit Rücksicht auf diese Doppelleitung nicht einverstanden sein können, wenn derselbe die Worte $\ddot{\gamma}_{i} \alpha \grave{\eta_{p}}$ I. 28 in Klammern setzt. Was aber die Einwendungen Alexanders betrifft, so liesse sich denselben wohl nur das eine entgegenhalten, dass ein Durchsehen durch die Mauern deshalb nicht möglich ist, weil die festen Theile so einander vorgelagert sind, dass die gerade Richtung des durch die feineren Poren dringenden Aethers nicht mehr aufrecht erhalten werden kann.) 
Die Auseinandersetzung (133, 28-38), dass durch jene Lehre vom Sehen wohl die Entstehung der Gestaltenbilder, aber nicht auch jene der Farben erklärt sei, wird noch dadurch eindringlicher, dass Alexander betont, es sei nicht einmal möglich, auf Grund der Analogie mit der Tastempfindung, wie sie von den Anhängern jener Theorie aufgestellt wird, die Entstehung der blossen Gestaltenbilder zu erklären. Denn (und damit wird der im vorigen Punkt zuletzt auseinandergesetzte Umstand eingehender beleuchtet) man ist zwar imstande, durch den tastenden Stock manche von den Gestaltungen der Dinge dadurch wahrzunehmen, dass derselbe über dieselben hingleitet, wie über das Convexe, oder dadurch, dass er die inneren Vertiefungen aufweist, wie bei dem Concaven, oder durch die Einhaltung der Mitte zwischen beiden zuletzt genannten, wie beim Geraden; aber dabei ist immer vorausgesetzt, dass der Stock seine eigene Gestalt nicht verändert, während das bei dem Sehmittel, der Luft, nicht angenommen werden könne, die sich vielmehr bei der fortlaufenden Berührung mit den Oberflïchen der einzelnen Körper und deren Begrenzungen je darnach immer neu gestaltet, so dass eine fortwährende Verschiebbarkeit der Theilchen jener Luft eintritt und dadurch das sichere, deutliche Sehen gehindert, dagegen die Verschwommenheit gefördert werde. (Wenn der Tastsinn und der Gesichtssinn sich vollkommen glichen, dann behielte Alexander mit diesen Einwendungen Recht; und relativ genommen behält er auch wirklich Recht, insoweit seine Polemik gegen die von seinen Gegnern vorausgesetzte Analogie zwischen den beiden genannten Sinnen sich richtet; aber trotzdem muss gesagt werden, dass dieselben nicht direct mit einander verglichen werden dürfen. Denn mittelst des Tastsinns erproben wir die äussere Gestalt der Dinge durch den Stock nur in der Weise, dass wir die Contouren derselben auf die Endigungen der Tastnerven einfach übertragen, so dass der Stock einzig das Mittel oder Werkzeug für die Empfindung ist. Beim Sehen dagegen fällt dieses Mittel oder Werkzeng weg, indem so ziemlich ganz unmittelbar der Reiz zum Angriff auf die Endigungen des Gesichtsnerven gelangt. Ueberhaupt lässt sich die Analogie zwischen beiden Sinnesgebieten bekanntlich schon deshalb nicht festhalten, weil einer derartigen Annahme die freilich 
auch wieder nur in einem gewissen Grade durchführbare Lehre von der sogenannten specifischen Energie der Sinne entgegensteht.) Ferner ergibt sich der Gesichtseindruck bloss am Ende des Objects, d. h. auf der dem Auge zugewendeten Seite. Und wenn man nun $(133,38-134,4)$ bedenkt, dass man nicht bloss von jenem dem Auge zugewendeten Theile des Objects Kenntnis erhält, sondern vom gesammten vor dem Auge befindlichen Gegenstande, so stellt sich uns die Frage entgegen, wie auf Grund jener Theorie auch das ganze Object wahrnehmbar wird, da man nach derselben meinen möchte, dass nur die dem Auge zugewendete Seite sichtbar sei. Nimmt man nämlich an, dass die Sichtbarkeit des ganzen Objects vermöge der Durchsichtigkeit der Luft möglich ist, so dass man auf diesem Wege das gesammte Object, welches von jener Luft durchzogen erscheint, vermöge der verschiedenen Anstoss im buchstäblichem Sinne erweckenden Theile der auf dem Objecte gelagerten Luft zu Gesichte bekommen kann, dann steht man mit dieser Annahme schon auf dem Boden einer neuen Theorie. (Alexander würde mit dieser Auseinandersetzung heutzutage als oberflächlich verschrieen werden, ein Vorwurf, dem er bloss deshalb entgeht, weil das Zeitalter jenes Philosophen noch nicht so geschult war, zwischen verschiedenen Erscheinungen auf diesem Gebiete zu trennen, wenn dieselben auch beim ersten Anblicke mit einander vermengt werden zu dürfen schienen. Dass man nämlich nur die Oberfläche eines Körpers sieht, ist eine bekannte Thatsache; eine unrichtige Ansicht jedoch hat Alexander insofern, als er meint, das Auge sehe nur die vordere Seite eines in die Länge gestreckten Körpers, selbst unter Anwendung der von ihm bekämpften Theorie. Denn dieselbe behauptet dies nicht, indem sie vielmehr durch die Annahme der Anspannung der Luft und Anpassung derselben an das Auge einerseits nichts darüber verlanten lässt, dass es nur die vordere Seite eines Körpers sei, welcher diesem Vorgang unterliegt. Man muss vielmehr aus den Worten am Anfange dieses Abschnittes entnehmen, dass die von Alexander bekämpfte Theórie in dieser Hinsicht ganz auf dem Boden der modernen Anschauung steht, wornach von jedem Punkte der dem Auge zugekehrten stereometrischen Objectsfläche ein Bild entsteht, ohne Rücksicht darauf, 
ob diese Punkte vorn oder hinten in dem Gegenstande sich befinden. Alexander scheint aber zugleich angenommen zu haben, dass die von ihm bekämpfte Theorie eine Verwechslung von Empfindung und Urtheil vorgenommen habe. Denn alferdings erkennen wir einen Gegenstand nach seiner vollen Ausdehnung erst dann, wenn wir auf Grund der durch die unmittelbare Sinnesauffassung gegebenen Momente des äusseren Eindrucks die Verbindung derselben unter einander und ihre endliche Zusammenfassung mit Rücksicht auf bereits bekannte Gestaltungen vornehmen, wie z. B. Wundt in seiner physiologischen Psychologie darthut. Man wird somit vorauszusetzen haben, dass Alexander mit seiner Zurückweisung eine Verrückung des Beweissatzes begeht, ohne dass damit gesagt ist, dass deshalb die von ihm bekämpfte Ansicht richtig ist. Aber noch eins ergibt sich vielleicht aus diesen Darlegungen. Man möchte nämlich bei der Lectüre der so eben angeführten höchst primitiven Anschauung über das Entstehen des Sehens von Körpern durch das Sichtbarwerden bloss der vorderen Enden der Objecte auf den Gedanken kommen, dass dieselbe auf populärer Auffassung beruht, welche denn auch vielfach in der ebenso primitiven Kunst der damaligen Zeit zum Ausdruck kam.)

Indem nun Alexander diese seine zuletzt vorgeführte Ansicht über die Sache mit der voraufgehenden verbindet, frägt er (134, 4-6), weshalb denn eine Durchsichtigkeit der festen Körper nicht stattfinde, da diese doch am geeignetsten seien, die Prägung der Objectstheile mittelst der Luft dem Auge zuzuführen, weil die letztere an ihnen bei diesem Vorgange einen festen Widerstand finde, während dies alles bei der Luft nicht stattfindet, welche dagegen wieder unter allen der durchsichtigste Körper sei. (Indem ich bemerke, dass hier die Theorie der Alten vom Ausgange der Lichtstrahlen vom Auge wieder aufzutauchen scheint, weil man sich sonst nicht recht vorstellen könnte, welches das Mittel dieser Prägung sein soll, füge ich nur noch hinzu, dass die ganze Polemik sich auf Grund des zu 133, 14-28 Bemerkten als ein Schlag ins Wasser erweist.)

„Wenn ferner“, fährt Alexander (134,6-9) fort, „die Luft durch Aufnahme der Einwirkung des Objects auf sie selbst die 
Gesichtsbilder erzeugt, so sei nicht zu erkennen, warum dieser Eindruck nicht auch nach Aufhören jener Einwirkung stattfinde, da man eine noch hinterher bleibende Wirkung ja auch bei anderen physikalisch-physiologischen Vorgängen wahrzunehmen vermag." Alexander will wohl sagen, dass, wenn der Gesichtseindruck von jener Einflussnahme einer Anspannung, eines Stosses und Schlages von seiten des Objects und seiner verschieden gestalteten Theile auf die umgebende Luft abhängt, so dass die letztere dadurch ein Gepräge erhält, welches, allein zum Auge fortgepflanzt, das Sehen der Gestalten bewirkt, dass in diesem Falle unmöglich davon gesprochen werden darf, dass die fortwährende Anwesenheit des Objects zum Zustandekommen des Gesichtsbildes erforderlich sei. Denn wenn es in letzter Linie bloss auf die "geprägte Luft" ankommt, dann darf das Object selbst, unbeschadet der zustande kommenden Wirkung, aus dem Gesichtskreis des sehenden Subjeets sich entfernen. (Wendet man das hier Gesagte auf den modernen Lichtäther an, dann wird man sagen dürfen, dass die Anwesenheit des Objects zum Zwecke des sinnenfälligen Sehens deshalb nothwendig erscheint, weil die fortdauernde Einwirkung des Objects auf den Aether deshalb erforderlich ist, weil die dadurch hervorgerufene continuirliche Wellenbewegung nur solange wirksam ist, als der Einfluss des Objects auf den Aether besteht, genau so wie ein Wasserrad nur solange in Bewegung ist, als es mit dem dasselbe treibenden Wasser in Contact sich befindet. Wenn also Alexander den Beweis für seine polemische Behauptung dadurch führt, dass er mit Zuhilfenahme einer Analogie auf alle anderen Fälle verweist, in denen nach Aufhören der Einwirkung des schlagenden, stossenden Gegenstandes oder Subjects in dem Geschlagenen, Gestossenen die bereits angefangene Wirkung des Schlages, Stosses fortdauert, auch wenn jener active, thätige Gegenstand nicht mehr vorhanden ist, so ist dagegen zu bemerken, dass diese Analogie eine nicht durchgehends zutreffende ist. Denn dass die Wirkung eines Schlages unter den angegebenen Bedingungen fortdauert, ist nur vom Standpunkt des Gefühls bei lebenden Wesen möglich, wogegen, wie wir sahen, rein physikalische Ursachen von kẹiner in dem hier vorgebrachten Sinne nachhaltigen Wirkung begleitet 
sind. Eine andere Frage ist aber die, ob man, nicht dennoch eine solche Nachwirkung voraussetzen muss, wenn man auf die sogenannten Nachbilder und auf die durch Reproduction erzeugten Vorstellungen Rücksicht nimmt. In diesem Falle wäre auch die Voraussetzung, nicht bloss das Argument des Alexander unrichtig, wornach in dem Falle kein Sehen mehr stattfindet, wenn das Object entfernt ist. Allerdings liesse sich noch darüber streiten, ob die angeführten Fälle von Gesichtsempfindung nach Entfernung des Objects noch ein eigentliches Sehen genannt werden können, aber der Grund, warum dann doch eine solche Nachwirkung noch besteht, liegt in der physiologischen Resonanz und nicht in einer ausserhalb des Gesichtsorgans noch nachwirkenden schwingenden Bewegung, wie von Alexander hier vorausgesetzt wird, einer Bewegung, welche nach dem rein physikalischen Charakter derselben wohl nicht weiter bestehen kann, wenn das dieselbe hervorrufende Object entfernt worden, ausser man nimmt nach der im vorigen Punkte von mir erwähnten Anschauung mancher Alten über den Ausgang der Lichtstrahlen vom Auge an, dass die von Alexander vorausgesetzten Schwingungen der Luft nach Entfernung des Objects ein zum physiologischen Nervensystem des empfindenden Subjects gehöriger Vorgang seien. In diesem Falle wäre aber damit eine nur sehr unvollkommene Erklärung der früher erwähnten Reproductionsvorgänge verbunden, wenn auch dieselbe als eine von jenem Philosophen für nothwendig gehaltene angesehen werden muss, da sie sonst nicht imstande waren, sich zu erklären, wie denu die Nachwirkungen der Sinnesempfindungen möglich sind, ausser man nimmt zu den erwähnten Mitteln seine Zuflucht. In jedem Falle ist diese von Alexander bekämpfte Ansicht interessant genug, um von dem Gesichtspunkt, den ihr hier Alexander entgegenhält, betrachtet zu werden, d. h. zu zeigen, wie die von unserem Scholiasten geprüfte Ansicht auch nach der Aufnahme desjenigen Punktes fähig ist, welcher nach der Voraussetzung Alexander's in derselben nicht mehr enthalten sein kann.)

Wenn dann Wasser über diejenigen Theile der Luft fliesst, in welchen $(134,9$ f.) sich der Grund zur Empfindung entwickelt, weil die letztere als der Erfolg der Bewegung betrachtet werden muss, 
die in jenen Lufttheilchen sich gebildet und schliesslich zu einer den Vertiefungen und Erhöhungen des Objects genau angepassten Ansammlung und Vertheilung jener Luft geführt hat, dann muss diese Anordnung einfach vernichtet werden, also dass man auch nicht mehr imstande ist etwas zu sehen, während wir doch vom Gegentheile uns überzeugen können, da ein fester Gegenstand, über den Wasser fliesst, immer noch sichtbar ist. (Gegen diesen Einwand lässt sich allerdings nichts vorbringen.)

Nicht passt auch zu der von Alexander bekämpften Theorie $(134,11-18)$ die Thatsache, dass eigentlich bei gerundeten Gestalten das Verhältnis des objectiven Gegenstandes zu der subjectiven Auffassung deshalb ein ganz anderer ist, als es zu jener Theorie stimmen kann, weil man dann concave Flächen im Auge convex und convexe concav sehen müsste; denn vermöge der Fortpflanzung jener Luftgebilde zum Auge ist es nothwendig, dass sie unausgesetzt in der ursprünglichen Lage bleiben, so dass, weil die dem Objecte aufliegenden Theilchen die innere Fläche des ganzen Sehraumes einnehmen, welcher eine ebenso innere, aber symmetrisch gegenüber liegende entgegensteht, aus geometrischen Gründen der nach Aussen gebogenen Fläche am Objecte eine nach innen gebogene im Auge entsprechen müsste. Mit Recht könnte dem die Thatsache gegenüber gehalten werden, welche aber Alexander ebenfalls wieder bekämpft, dass nämlich bei der Tastempfindung etwas Aehnliches stattfinde, indem auch bei der Betastung von gekrümmten Flächen durch die hohle Hand letztere concav eingebogen erscheint, wenn sie eine convexe Oberfläche befühlt. (Wenn wir einen Augenblick bei dieser Thatsache verweilen, so können wir uns nur an jene viel erörterte Frage erinnern, wie man trotz des umgekehrten Netzhautbildes die Gegenstände der Aussenwelt aufrecht sieht. Ich will hier im Vorbeigehen bloss bemerken, dass man, um zu erkennen, ob ein Gegenstand aufrecht ist oder nicht, sich des Urtheils bedienen muss, also dass man bereits nicht mehr auf dem Boden des reinen Empfindens, der Gesichtsempfindung steht.) Alexander bemerkt hierauf, dass gegen die Annahme von der dem Auge analogen Empfindungsfähigkeit der Hand die Thatsache spreche, dass man, um hohle oder gewölbte Gegenstände 
durch Zeichnung oder Malerei darzustellen, - nur ebener Flächen

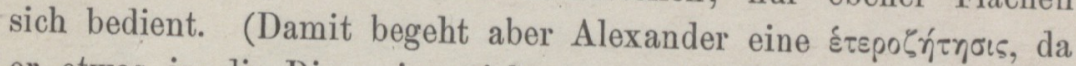
er etwas in die Discussion zieht, was ohnehin auch von seinen Gegnern nicht bestritten wird, ohne dass dadurch der Beweis auch nur im geringsten gefördert wäre.)

Ein ähnliches Verhalten erscheint auch rücksichtlich der Reflexion des Lichtes vom Spiegel gegen die in Rede stehende Theorie zu sprechen $(134,18-23)$. Denn wenn man bedenkt, wie es in der Natur der Sache gelegen wäre, dass die gekrümmte Oberfläche des Lichtkegels, welcher auf einen Spiegel fällt, von letzterem in einer Fläche reflectirt werden müsste, welche der Krümmung der einfallenden Kegelgrundfläche gerade entgegengesetzt ist, oder dass die gekrümmte Fläche nach der Reflexion zu einer ebenen geworden sein müsste, dann kann von einer Identificirung des ersten und des Spiegelbildes gar keine Rede mehr sein, weil sich die beiden nicht mehr glichen. (Es scheint aber, dass auch hier Alexander die Thatsache der Symmetrie des Bildes und Objectes ausser Betracht gelassen hat.)

Indem sich ferner Alexander auf das bereits $133,4 \mathrm{ff}$. Bemerkte beruft, sagt er im Anschluss an den vorigen Punkt noch einmal (134, 23-26), dass es unmöglich sei, dass die Integrität des Bildes gewahrt werde, wenn dasselbe von einem Spiegel reflectirt erscheint.

Endlich sei nieht abzusehen, warum wir nicht auch die unmittelbar vor den Augen befindlichen Dinge sehen, weil ja auch $(134,26 \mathrm{f}$.) auf diese das (von Alexander bekämpfte) Princip angewendet werden müsste, umsomehr als das Bild dieser Gegenstände im Auge wegen-der grossen Nähe noch viel deutlicher werden kann. (Natürlich hätte Alexander anders gesprochen, wenn er die physiologische Einrichtung des Auges, insbesondere mit Rücksicht auf das Vorhandensein der Linse genauer gekannt hätte.) 
(Sonderabdruck aus dem Archiv für Geschichte der Philosophie VIII. Band 4. Heft. 1895.)

Druck von Georg Reimer in Berlin. 


\section{Figh $22 \cdot \operatorname{Man} \cdot \overline{97}$}

sheor $a$. 4II

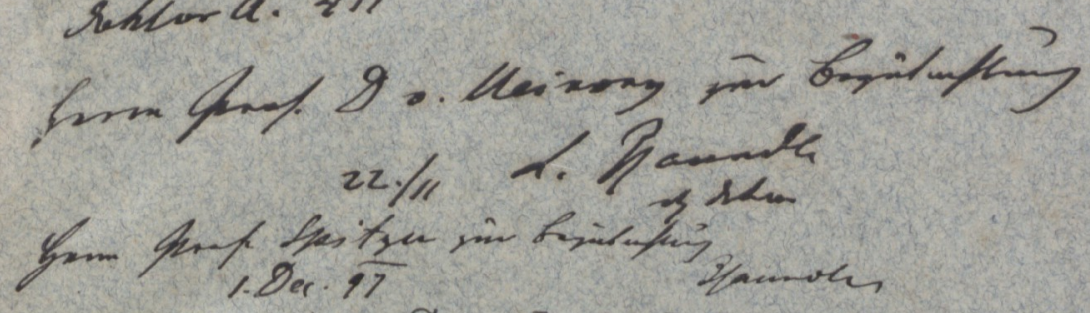

\section{Sonderabdruck}

aus dem

\section{Archiv für Geschichte der Philosophie}

in Gemeinschaft mit

Wilhelm Dilthey, Benno Erdmann und Eduard Zeller

herausgegeben

von

Ludwig Stein.

B a n d IX, H e f t 2 . 



\title{
Die Polemik Alexanders von Aphrodisia gegen die verschiedenen Theorien des Sehens.
}

\author{
Von
}

\$oh. Zahlfieisch in Graz.

III.

1) Ueber das Sehen, durch welches Objectsbilder zum Auge gelangen.

Zunächst wäre es, meint Alex. 134, 30-32 unmöglich, dass nicht bei der Annahme des Ausgangs sovieler Objectsbilder, da hiedurch für jedes sehende Auge ein solches gegeben wäre, eine rasche Abnützung dieser Emanationskraft eintrete, worin Alex. natürlich mit Rücksicht auf die von ihm bekämpfte Theorie Recht, insofern aber Unrecht hat, als das Object kein leicht auflösbarer Gegenstand ist, wie etwa Rauch oder Nebel, sondern immer, eine entsprechende Beleuchtung vorausgesetzt, auf das Auge zu wirken vermag.

Wenn man aber die Voraussetzung macht, dass den von aussen zum Auge gelangenden siò $\omega \lambda \alpha$ von seiten des letzteren zuhilfe gekommen würde. (wie Alex. sich dies denkt, lässt er vermöge seines

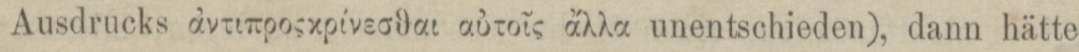

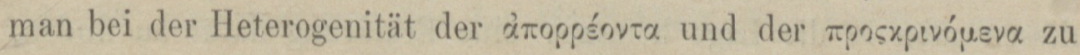
gewärtigen, dass eine entsprechende Vereinbarung nicht stattfinde $(32-36)$. Es ist ein kleines Stück Wahrheit in dieser Annahme, 
weil man selbst auf dem Gebiete der Sinnesempfindung den Satz:

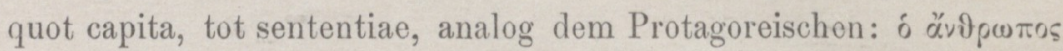

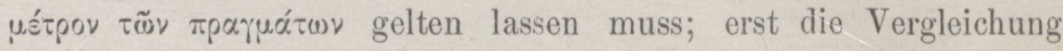
auf der Basis der Erziehung und des gesellschaftlichen Verkehrs lässt uns jene Seiten in unserer Sinnesempfindung hervorkehren, welche als allgemeiner Erfahrungsschatz der Menschheit von Wichtigkeit sind.

Ferner wäre $(36-135,2)$ die Nothwendigkeit des fortwäh-

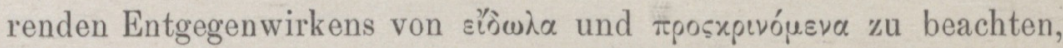
so dass die bei diesem Vorgang entstehenden jeweiligen Bilder selbst sich gegenseitig hindern und auf solche Weise ein Mischbild zustande käme, das nicht unähnlich unseren modernen Interferenzerscheinungen wäre (natürlich käme das $\mathrm{zu}$ den beiden ersten Puncten Erwähnte hier in verstärktem Masse zur Anwendung).

Wie ist weiter eine Abschätzung der Entfernung des Objects vom sehenden Auge möglich, wenn man sonst weiter nichts sieht

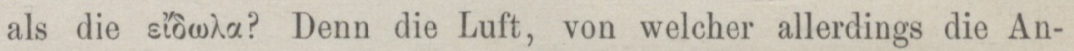
hänger jener Lehre voraussetzen, dass sie von den siồ $\alpha$ vor ihnen her getrieben werde, ist nicht empfindbar, wenn man auch annehmen wollte, dass durch deren Quantität die Entfernung abgeschätzt werde. (Vielleicht ist auch Alexander in den Fehler verfallen, in den Beweisen seiner Gegner eine Trennung der einzelnen Beweismomente nach gleicher Art vorzunehmen, wie dies Aristoteles bei der Polemik gegen Platon so gerne thut, indem er nach dem Grundsatze: Divide et impera! vorgeht. Denn jedenfalls müssen wir von unserem modernen Standpunct hinzufügen, dass zur Abschätzung der Distanzen neben der Convergenz und Accommodation noch eine Menge anderer Umstände mitwirken, unter welchen nicht der letzte die Erfahrung des Subjects selbst ist.) 2-5.

Alex. fährt fort, es sei keine Möglichkeit vorhanden, dasjenige Element zu finden, in welchem die Gewähr liegt, dass dadurch diese Luft (ihrer Grösse nach) abgeschätzt werde (5). Ausserdem sei es nicht möglich, einen Modus sich zurecht zu legen, mittelst dessen man in die Lage komme, eine Erklärung dafür zu finden, dass man bei raschem Aufblicken auch sogleich das Bewusstsein von einem in der Ferne liegenden Gegenstande habe (5 f.). (Alex. 
meint wohl im Anschluss an das Vorige, dass es nicht so leicht sei, sich sofort und auf der Stelle eine Vorstellung von der Menge jener Luft zu machen, die vor den siówi $\alpha$ einhergetrieben wird, und deren Quantität der Entfernung des Gegenstandes entsprechen soll. Doch ist dieser Einwand insofern nichtig, als der menschlichen Psyche eine. wunderbare Kraft der Uebung im Abschätzen von was immer für Einwirkungen auf sie selbst eignet, da wir sogar imstande sind, durch unmittelbares Bewusstsein und rasches Abwägen der Erscheinungen desselben die theoretisch so relativ compliciert sich präsentierenden psychophysischen Gesetze in Wirklichkeit zu übertragen und dadurch bei jedem Thun zu verificieren.) Näheres zu beiden Puncten s. unten Z. $11 \mathrm{ff}$.

Schwierigkeiten bereite auch die Auffassung der geometrischen Gebilde, weil man erstlich zu wenig Raum habe, um ihre Gestalt abschätzen zu können; denn hier muss bemerkt werden, dass die kleine Ausdehnung des Augensterns, welcher kaum die Grösse des

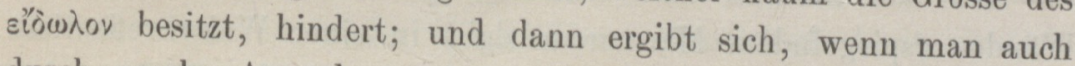
durch rasche Augenbewegungen die einzelnen Theile des Objects nach einander in das Sinnesorgan sozusagen hereinzieht, dass aus diesem Contiguum ein Continuum nicht erzielbar ist. (Vor allem hat man zu bemerken, dass dem Alex. die Thatsache unbekannt sein musste, dass das Bild des Objects auf der Netzhaut ein verkleinertes ist; dann aber hat Alex. eine Wahrheit gelassen ausgesprochen, ohne sie für sich zu verwerten. Denn es ist in Wirklichkeit ein Problem, wie durch die in der That nur Contigua darstellenden Bildpuncte ein continuierliches Bild des Objectes erzeugt wird. Nebstdem muss bezüglich des Textes erwähnt werden, dass ich trotz der vom Herausgeber citierten Parallelstelle keinen Grund einsehe in 12 ein oi $\chi \grave{i}$ einzuschalten, da es sich um die Herstellung eines Continuums zwischen oft gesehenen nämlichen oder verschiedenen Puncten handelt, von welch letzteren jeder nur Einmal gesehen wird.) (6-14.) Vgl. unten Z. 139, 14-17.

Im Anschluss an das zuletzt Gesagte wendet Alex. ferner (14-18) ein, dass man bei der Möglichkeit, dass bei dem zuletzt von ihm geschilderten Verfahren in dem Objecte nicht zusammengehörige Puncte bei dem Bemühen, das demselben entsprechende 
Bild zu gewinnen, nun auf einmal auseinanderfallen, so dass man nicht mehr das entsprechende, sondern ein ganz anderes Bild erhält, nie in der Lage sei die Bürgschaft dafür zu besitzen, dass man auch richtig gesehen habe. (Das fest fixierte Bild auf der Netzhaut kann jedoch von einem ruhenden Gegenstande trotz der fortwährenden methodisch geübten Augenbewegungen nur ein dem Object entsprechendes Bild liefern, was Alex. bei dem Mangel der Anatomie des Auges freilich nicht wissen konnte.)

Indem unser Verfasser ferner immer wieder von der leichten Veränderbarkeit der Theile jenes siò nicht begreifen, wie dieses seine ursprüngliche Gestalt beizubehalten vermag, er weiss nicht, wo dieselben hingerathen, in welchen Tiefen sie verschwinden, wer sie zu einem einheitlichen Ganzen verbinde. (Selbstverständlich muss auch darauf das Nämliche erwiedert werden wie zum vorigen Puncte.)

Wie soll man es dann erklären, dass die siò $\omega \lambda \alpha$ von glatten Wänden (spiegelndem Marmor u. dgl.) in einer Weise reflectiert werden, dass man keine Veränderung der Gestalt des Bildes wahrzunehmen imstande ist, ohne dass das Gesichtsorgan dabei thätig ist? Und wie erklärt man sich dasselbe bei spiegelndem Wasser? Wie lässt sich ein feststehendes und so deutliches Bild im Spiegel erklären, wenn man bedenkt, dass die siôw strömen sollen? (24-29.) (Die von Alex. aus der Annahme seiner Gegner gefolgerte Dehnbarkeit und Veränderlichkeit der siò $\omega \lambda$. muss natürlich gemäss der Regelmässigkeit, die in dem Gesetze der Reflexion liegt, zurückgewiesen werden. Und das Bleibende des $\varepsilon^{\prime} \hat{c} \omega \lambda$ ov im Spiegel ist nur eine Folge der Fortwirksamkeit der Lichtquelle.)

Warum, meint Alex. ferner, bleiben die siô $\omega \lambda \alpha$ im Spiegel unveränderlich, da sie doch vermöge der ihnen zukommenden Beweglichkeit sich immerwährend ändern sollten (so dass man vielleicht ein allmähliches Verschwinden derselben supponieren könnte)? (29 f.) (Die Erklärung dafür ist schon gegeben.)

Und wenn man den siồi $\alpha$ in Consequenz der von Alex. seinen Gegnern zugeschriebenen Lehre annehmen wollte, dass sie auch dann bestehen sollten, wenn gleich nur kurze Zeit, wenn das 
sehende Subject sich entfernt hat, und wenn man sieht, $-\bullet$ dass dies nicht stattfindet, so muss gefragt-werden, worin diese Inconsequenz

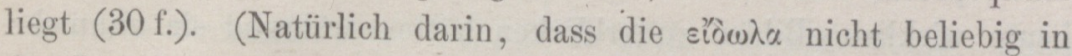
der Luft herumfliegen, sondern an ein Object gebunden sind, mit dessen Entfernung auch das Verschwinden des Bildes verbunden ist.)

Warum sind die siò $\omega \lambda \alpha$ in der Tiefe des Spiegels und nicht auf der Oberfläche? (31f.) (Dieser Einwand ist gewiss erklärlich, weil von den siôwì nicht erwartet werden kann, dass sie eine Bewegung durch das harte Spiegelglas hindurch zu machen imstande sind, vielmehr der Gedanke nahe liegt, dass dieselben da auflagern, wo sie auf ein hartes, Widerstand leistendes Object treffen. Doch hat die neuere Physik nachgewiesen, dass das Bild erst auf der Netzhaut entsteht und nur hinter den Spiegel projiciert wird.)

Man kann sich ferner, auch wenn vorausgesetzt würde, dass sich die noch etwa in der Luft befindlichen Gegenstände durch die siô $\omega \lambda \alpha$ hindurchbewegen, ihre Standhaftigkeit nicht erklären, da sie doch so leicht verschiebbare Theilchen besitzen (135, $32-136,2)$. (Und wenn wir nun wissen, dass in der That das Vorschieben eines anderen Objects genügt, um das früher gegebene verschwinden zu machen, so wäre die Unmöglichkeit davon erklärt;

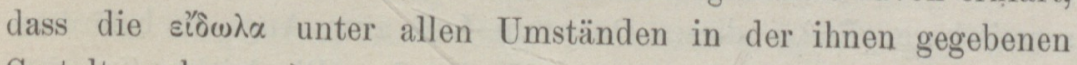
Gestalt ausharren.)

Und sollte man dieses Durchgehen beim Verharren der Gestalt jener sไô $\omega \lambda \alpha$ erklären wollen, dann müsste man einen leeren Raum supponieren, durch welchen dieses Durchgehen ermöglicht wäre $(136,2$ f.). (Da von einem Durchdringen nicht, sondern nur von einem Verdecken gesprochen werden kann, wie wir im vorigen Puncte gesehen, so entfällt natürlich diese weitere Einwendung des Alex., welche wegen ihres Anklangs an die Verwerfung eines absolut Leeren durch Aristoteles besonders bemerkenswert erscheint.)

Und wenn der Wind weht, so könnte man nichts deutlich sehen, nach dem zu 135, 32-136, 2 Bemerkten (136, 3-5), wozu man die zu jener Stelle von mir gemachte Bemerkung vergleiche.

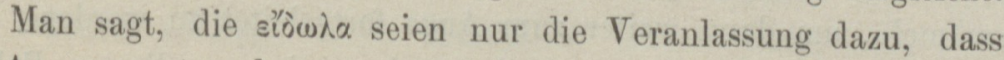
das Auge erregt und angeregt wird, um in die Ferne schauen und die daselbst befindlichen Objecte sehen zu können. Aber dabei 
bewegen sich diejenigen, welche derartiges behaupten, in einem Widerspruch, insofern man in den siô Leute wirkliche Bilder zu erblicken hätte, während sie nunmehr behaupten, dass sie durch Erregung des Auges das Sehen erst veranlassen sollten $(5-11)$. (Hatte Alex. das, was er hier den

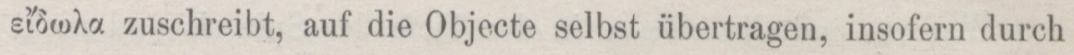
das beleuchtete Object das Auge wirklich angeregt wird, dann hätte er nicht Recht. Der Tadel, den Alex. ausspricht, bewegt sich also einerseits auf der Basis der Annahme, dass was man sieht, nicht das Object, sondern das stồ die Ferne unmöglich wäre, wenn das vom Object hervorgegangene

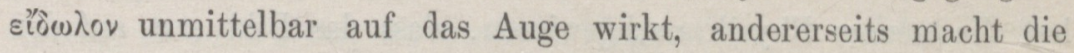
Voraussetzung, dass es etwas Anderes sein müsse als das Object, was unmittelbar wahrgenommen wird, dem Urheber jener Ansicht

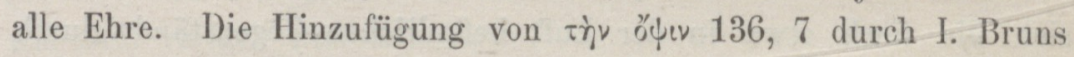
finde ich trotz der Belegstelle für nicht nothwendig.)

Die Möglichkeit in die Ferne zu sehen (6) wird durch die Annahme nicht vergrössert, dass es dem Auge möglich ist, die

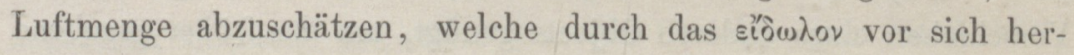
getrieben wird, weil man sich nicht vorstellen kann, wie das leicht zerreissbare s'ố vermag $(11-16)$. Vgl. ob. zu 135, 2-6. (Die leichte Zerreissbarkeit des s'ồंगे entfällt, wenn man sich von der Annahme solch körperlicher Zwischengebilde emancipiert, obschon die Voraussetzung eines allgemeinen Aethers, der in unserem Falle zum Lichtäther determiniert ist, es mit sich brächte, dass man eine Körperlichkeit vom Object zúm Auge bei der. Entstehung des Bildes annimmt.)

Wenn man annimmt, dass durch die wiederholt wirkende Kraft des Anprallens der Luft von den siòwì $\alpha$ her am Auge dasselbe geschädigt werden könnte, so wäre es vielleicht möglich, dieser Schwierigkeit dadurch zu entgehen, dass man eine letzte Schicht vor dem Auge voraussetzt, in welcher die Luftwogen sich sammeln, um dann weiter zum Auge fortgeleitet zu werden. Nur lässt sich kein Anstoss ausfindig machen, der zu diesem Behufe von dieser letzten Schicht ausgienge $(16-21)$. (Alex. findet hier 
bereits Elemente vor, die nicht mehr weit von der früher angesehenen Undulationstheorie entfernt sind.)

Die ebenfalls oben 135, 2-6 offen gelassene Frage, woher das so leicht zerreissbare siò $\omega \lambda$ ov die Kraft nehme, um eine so grosse Luftmenge vor sich herzutreiben, wird als weitere Instanz gegen Alexanders Widersacher aufgestellt (21-24). (Es hat auch heute noch für uns eine Schwierigkeit, das Nebeneinanderbestehen von Aether- und Luftwellen bei der Entstehung von Licht und Schallempfindungen zu erklären.)

Die Farblosigkeit der siồì und der Umstand, dass bei der nicht selten vorkommenden Grösse derselben das Auge zu klein ist, um die durch jene $\varepsilon \iota_{\partial} \omega \lambda \alpha$ dargestellten Gestalten aufzunehmen, erklären allzuwenig die Möglichkeit des farbigen und geometrischen Sehens (24-28). (Die Kleinheit des Bildes auf der Netzhaut, gepaart mit der von der Psyche ausgehenden Subjectivität der Anschauung macht es möglich, für das geometrische und räumliche Sehen eine Grundlage zu gewinnen. Was aber das Gefärbtsein anbelangt, so spielen hier nicht bloss die Eigenschaften des bei der Darstellung der $\varepsilon \hat{i} \hat{\delta} \omega \lambda \alpha$ vollständig ausser acht gelassenen, in farbige Bestandtheile zerlegbaren Lichtes mit, sondern auch die chemischen Reagentien, Licht- oder Sehpurpur u. dgl., Dinge, welche damals durch eine auch noch bei Goethe vorkommende Mischung des ursprünglich weissen Lichtes mit verschiedenen Dunkelheitsgraden ihre, wenn auch unklare Deutung erhielten.)

2) Gegen die Annahme derjenigen, welche vom object und vom Auge Ausflüsse annehmen.

Die Voraussetzung, dass (bei der verschiedenen Entfernung der Objecte vom Auge) ein nicht bei jedem Sehen gleichzeitiges Zusammentreffen der beiderseitigen Ausflüsse denkbar ist, macht es unerklärlich, dass wir doch imstande sind, nähere und fernere Gegenstände zu gleicher Zeit zu sehen. Ausserdem müsste sowohl die Lebhaftigkeit des Eindrucks verschwinden, welchen das Object zu machen vermag, wenn allmählich immer mehr und mehr Ausflüsse demselben entströmen, als auch die Sehkraft des Auges aus dem gleichen Grunde geschwächt werden $(136,29-137,22)$. 
(Wenn von 2 Ausflüssen die Rede ist, von denen der eine vom Objecte, der andere vom Sabject ausgeht, dann lässt sich das allerdings mit der Lichtquelle des gesehenen Gegenstandes und mit der Kraftquelle des sehenden Auges vergleichen. Aber es hängt die von Alex. bekämpfte Ansicht mit einer Anzahl von Fragen zusammen, welche ihm nicht bekannt sein konnten, vor allem mit der Frage der Schnelligkeit des Lichtes. Nebstdem will ich nur das Problem hier berühren, was denn eigentlich als gesehen von mehreren Philosophen vorausgesetzt angenommen werden sollte, ob der blosse äussere Eindruck, die unbestimmte Gestalt und Farbe, mit Einem Worte die allgemeine Sinnesempfindung, oder die genaue Erkennung des Objects. Wir wissen, dass letzterer Umstand durch die Zeitabschätzung Gegenstand psychophysischer Demonstration ist.)

Der folgende Einwand ist aristotelisch-dialektischer Natur und und ähnelt den Aussprüchen, dass die Natur nichts umsonst thue, und dass aus dem uns Bekannten das an sich Bekannte abgeleitet werden muss. Denn Alex. bemerkt (23-25), dass man unter der Voraussetzung, dass beide Ausflüsse sich auf gerader Linie bewegen, zum Zwecke der Erklärung des Problems nicht gezwungen sei, zu einer beiderseitigen ḋ róppoıx seine Zuflucht zu nehmen, weil eine auch genüge (als ob die Natur sich vorschreiben liesse, was sie zu thun hat).

Der von Alex. bekämpfte Philosoph musste aber angenommen haben, dass der vom Objecte her sich erhebende Ausfluss den

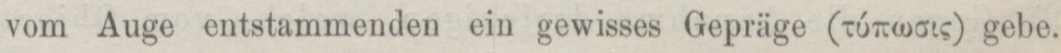
Nun frägt Alex., weshalb denn dabei eine Veränderung (vermöge des Aufeinandertreffens der beiden Ströme) vorhanden sei (25-28). Man denkt unwillkürlich an eine Art elektrischer Entladung an der Berührungsstelle beider Ströme.)

Die Nothwendigkeit, mittelst der siò $\omega \lambda \alpha$, oder besser, der gefärbten siồ $\omega \lambda \alpha$ das Zustandekommen des Gesichtsbildes zu erklären (vgl. 136, 24ff.), zeigt den Weg für die Unmöglichkeit der hier vorausgesetzten Annahme. Denn entweder ist der Grund der Gesichtsempfindung das siồiov, und dann lässt sich keine Farbe erkennen, oder man erkennt zwar die Farbe, kommt aber dann 
nicht dazu, einen Ausweg zu finden, wie neben der Farbe auch die Gestalt wahrgenommen werden kann. Und nebs̈tbei weiss man micht die Angriffsart der von aussen an das Sehorgan tretenden Einflüsse anzugeben (28-34). (Die Frage über die Beschaffenheit der an das Auge gelangenden Einflüsse war vom Standpunkte Alex.s bei Festhaltung der von seinen Gegnern gebotenen Anschauungen freilich am Platze, weil er nicht imstande war, die Möglichkeit einzusehen, wie aus blossen Aus- und Einflüssen eine subjective Empfindung so complicierter Art sich ergeben soll, wie die des Gesichts eine ist.)

Unter der Voraussetzung, dass die vom Auge ausgehende ¿̀mópposa Licht ist, müsste ja der eine des Anderen àmóppoia sehen, wenn Dunkelheit eintritt (beim Tageslicht sieht man sie ja ohnehin nach jener von Alex. bekämpften Hypothese), in ähnlicher Weise, wie wenn man auch in einer dunkeln Kammer eine Lichtempfindung hat, wenn nur durch eine Lïcke ein Strahl auf einen Augenblick hereinkommt $(34-138,2)$. (Der Einwand ist nicht übel, wenn man überhaupt die Voraussetzung gelten lässt, dass nur eine einseitige àmóppoıa, die vom Auge herkommende, vorhanden ist und an sich schon einen Lichteffect bewirkt.)

Dem nächsten Einwand, dass unter der hier gemachten Voraussetzung das Licht als Körper gegeben sein müsste, widmet Alex. ein eigenes Capitel.

Das Licht ist nicht Feuer.

Hiebei kommen 3 Möglichkeiten in Betracht, entweder ist das Licht einfach Feuer a) oder Ausfluss des Feners (Glanz) b) oder eine besondere Art des Feuers c) (vor xai tpítov muss bei Ivo Bruns 138, 5 Komma stehen). - a) Nun gibt es aber, sagt Alex., verschiedene Empfindungsintensitäten für das Licht, jedoch nicht für das Feuer (welches immer mit der gleichen Kraft brennt). Sollte man auch von einer grösseren Stärke des Feners sprechen, dann heisse man dies Flamme, und nicht mehr Feuer schlechthin (138, 3-9). (Mit dem gleichen Argumente hätte Alex. irgend ein anderes Element als mit dem Lichte nicht zu verwechseln heranziehen können, weil das Licht sich bei der Gesichtsempfindung, die uns die entsprechende Kenntnis was immer für eines Gegen- 
standes vermittelt, nur als Eigenschaft, nicht als fassbares Element entgegen zu treten scheint. Und um es kurz zu sagen, können wir von Licht nicht in dem Sinne sprechen, als sei es ein selbständiges Ding, sondern das Licht kommt erst durch Zuhilfenahme unseres Empfindungsorganes zustande.)

Dass Licht nicht Feuer ist, lässt sich, sagt Alex., dadurch erweisen, dass man findet, es brenne wohl das Feuer, sowie es erwärmt, aber nicht das Licht; es könne kein Feuer im Wasser oder im Eise existieren, wohl aber das Licht (9-12). (Nun hat aber Alex. nicht bedacht, dass das Feuer eines entsprechenden Materials bedarf, durch dessen Hilfe es fort und fort ernährt wird, des Brennmaterials, sei es $\mathrm{Holz}$ u. dgl. oder Sauerstoff; das ist nun beim Lichte nicht der Fall, da es vielmehr deshalb auch im Innern der Körper gesehen werden kann, so dass dieser Vergleich Alex. zwischen Licht und Feuer bedeutend hinkt.)

Und wenn man auf die Bewegung des Lichtes und Feuers Rücksicht nimmt, meint Alex. (12-14), so sehe man einen Unterschied zwischen beiden darin begründet, dass dieses nur in der Richtung nach oben, jenes auch in der nach unten sich bewegt. (Alex. setzt hier die naturgemässe Bewegung als Unterscheidungspunct voraus; allein er wusste nicht, dass dies nur in unserer Erdatmosphäre so sei, während man das Sonnenfeuer auch nach abwärts brennen sieht und dies überhaupt von der Stelle der Erde abhängt, in welcher man auf derselben sich befindet.) -

b) Wenn das Licht bloss ein Ausfluss des Feuers wäre, so müsste dasselbe nur dann existieren, wenn Feuer vorhanden ist, wogegen aber das Gegebensein von Licht, wie bei dem Glühwürmchen, eine Instanz bilde (14-16). (Die in moderner Zeit gefundene Erklärung für das Zustandekommen des objectiven Lichtes, welches das Product elektrischer Kräfte sei, bildet eine für die Entstehung des Feuers in gleicher Weise gegebene Basis.)

Unter der nämlichen Voraussetzung müsste man aber auch annehmen, dass mit der Entfernung der Quelle für jene Ausflüsse noch nicht die Ausflüsse selbst aufgehoben sind, sondern dass sie nach Analogie ähnlicher Vorkommnisse eine Zeitlang noch nachher fortbestehen (ohne dass man dies beim Feuer wahrzunehmen ver- 
mag; denn nach Entfernung des Feuers hört auch der Glanz auf) (16-18). (Nun liesse sich aber fragen, welche Analogien Alex. hier meint. Setzt er z. B. voraus, dass man nach Untergang der Sonne noch ihr Leuchten in den Wolken wahrnehme, dann müsste das Licht ein Reflex sein, was es jedoch nicht ist.) - Aus dem Umstande der Nothwendigkeit, dass immer die Quelle des Lichtes vorhanden sein müsse, folgert Alex. weiter, dass das Licht selbst ungemein leicht zugrunde geht; und da möchte man nun glauben, dass das Licht der Gegenstände, welche in unausgesetztem Glanze erstrahlen, vermöge der durch seine Beständigkeit bewiesenen Stärke mit Rücksicht auf seine Herkunft aus dem Feuer diese Gegenstände selbst vernichtet $(18-22)$. (So fein ausgeklügelt der Gedanke ist, so wenig kann er an der Sachlage ändern, weil man über die Dichtigkeit des angeblichen Ausflusses des Feuers nicht im Klaren sein kann; denn wenn diese geringe Dichtigkeit hinreicht den Lichteffect zu erzielen, dann braucht auch ein längeres Anhalten derselben nicht das im Gefolge zu haben, was Alex. hier voraussetzt.)

Die Art der Lichterhaltung bei den Leuchtkäferchen ist bei dieser Annahme gleichfalls unbekannt (23). (Natürlich! denn dem Alex. konnte die wahre Ursache der Phosphorescenz nicht bekannt sein.)

Die Eigenthümlichkeit des Lichtes als eines Körpers hat ferner die Unmöglichkeit im Gefolge, das Dilemma zu entscheiden, nach welchem man entweder 2 Körper in Einem Raume anzunehmen hat (die durchsichtigen Körper und das Licht) oder die Voraussetzung, dass das Licht ein Körper sei, aufgeben muss. Und dieses erhellt aus folgender Discussion. Das Licht geht durch Glas u. dgl. Körper; wenn also das Licht Feuer oder ein anderer solcher Körper ist, dann kommt man zum obigen Schlusse (23-29). (Dass Feuer durch einen Körper geht, ist zwar nicht unmittelbar richtig; aber wenn durch Feuer Körper verzehrt werden, und wenn die Wärme durch Körper hindurchdringt, dann liesse sich durch eine Art Analogie die Möglichkeit erweisen, dass das Licht doch eine Art Körper ist, ähnlich dem elektrischen Fluidum, welches ebenfalls Körper durchdringt.) 
Es müsste aber auch der Schatten, entsprechend dem Lichte, ein Körper sein, was gegen die allgemeine Anschauung ist, oder man müsste den Schatten für unkörperlich betrachten, so dass (nach dem Grundsatze, dass Entgegengesetzes unter Einem Princip steht - ein bei Aristoteles geläufiger Satz $\rightarrow$ ) auch das Licht kein Körper mehr sein könnte (29-31) (offenbar beruft sich für den ersten Fall Alex. auf eine Annahme, die erst des Beweises bedarf, und im letzteren Falle steht die Regel, welche er voraussetzt, noch in Discussion).

Bei dem Zusammentreffen von Licht und Schatten könnte man fragen, ob das erstere durch letzteren nicht in seiner Existenz gestört werde, so dass dann Licht, Schatten und zugleich Luft in einem und demselben Raume wären. Es könnte aber auch angenommen werden, dass bei der Aufnahme des Lichtes von seiten des Schattens der letztere dahin geht, woher er gekommen, nämlich zur Quelle des Lichtes, Sonne, Gestirne und Feuer. Dann müsste aber der Schatten auf diesen Körpern wohnen $(31-36)$. (Die Möglichkeit verschiedener Träger mehrerer Naturgewalten in einem und demselben Raume hat offenbar Alex. ausser acht gelassen, da er nicht wusste, dass die den Raum erfüllende Luft ein chemisch zusammengesetzter Stoff ist, dessen Elemente als jene Träger der Naturkräfte Licht, Schall, Elektricität, Wärme u. s. w. angenommen werden müssen. Und was den Schatten betrifft, so muss man denselben nur als einen besonderen Effect des Lichtes, nicht als Lichterscheinung selbst betrachten.)

Die erstere Annahme des vorigen Punctes, dass durch den Schatten oder durch das Dunkel das Licht aufgenommen wird, führt zu dem absurden Resultate, dass entgegengesetzte Eigenschaften zu gleicher Zeit an dem nämlichen úmoxsíusvov sich befinden $(138,36-139,1)$. (Aber sollte es nicht möglich sein, dass der Schatten vom Lichte einfach verdrängt wird?)

Das Zusammentreffen des körperlichen Lichtes mit der Luit hätte den Umstand im Gefolge, dass 2 Körper sich gegenseitig durchdringen, wenn man nicht lieber der Luft Poren gibt, durch welche das Licht geht. Im letzteren Falle (der erstere trägt ohnehin sein Absurdum auf der Stirne) müsste man in die Poren (da 
ein wirklich Leeres nicht existiert - das ist Aristotelische Annahme) einen Stoff hineinversetzen, welcher dtinner ist als das Licht, weil er diesem letzteren nachgibt, während man doch anzunehmen hat, dass es keinen dünneren Stoff mehr gibt (letzteres wohl nach populärer Anschauung). Und wenn man gelten lässt, die Poren seien mit Feuer erfüllt, dann könnte man sich das Entstehen des Dunkels nicht erklären, weil das Feuer nur erwärmt und erleuchtet, während dies von seiten des Dunkels nicht geschieht. Ausser dem angegebenen Grunde für die Nothwendigkeit des Erfülltseins der Poren durch irgend einen Stoff hebt Alex. noch hervor, dass die Luft wegen ihres unter jener Voraussetzung anzunehmenden Durchzogenseins von leeren Poren dünner. wäre als das consistente Dunkel, welches ja als Körper vorausgesetzt ist, zumal der Nacht, in welcher man keine dünnere, sondern eine dichtere Luft spürt als am Tage, so dass dann nicht mehr das Licht als der Zertheiler der Luft, sondern umgekehrt die Luft als der Zertheiler des Lichtes gälte (139, 1-14). (Die Voraussetzung von dem Stoff in den Poren der Luft deckt sich beinahe mit unseren modernen Anschauungen über die chemischen Moleküle.)

Unter der zuletzt gemachten Voraussetzung könnte man eine Instanz gegen die in Rede stehende Annahme darin finden, dass man ungleichmässig beleuchtete Flächen sehen sollte, wenn die Lichtporen durch Luft unterbrochen sind (14-17). (Und das ist auch der vernünftigste Einwand gegen jede Atomtheorie, welche letztere schon von Aristoteles bei verschiedenen Gelegenheiten zurückgewiesen wurde. Vgl. meine in der Zeitschr. f. Philos. u. philosoph. Kritik erscheinende Abhandlung: Zur Kritik der Aristotelischen Metaphysik, Band 105, S. 218 ff. u. oben zu 135, 6-14.)

Im Folgenden (17-19) bringt Alex. einen ähnlichen Einwand vor, wie oben zu 137, 34-138, 2.

$\mathrm{Zu} 19-24$ ist $\mathrm{zu}$ bemerken, dass die hier von Alex. vorausgesetzte Schnelligkeit des Lichtes ein ganz richtig gestelltes Postulat ist.

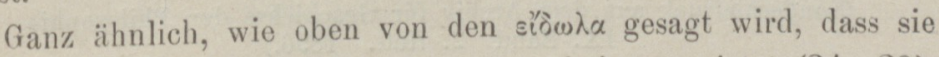
sich beim Wehen des Windes bewegen, heisst es jetzt (24-28), 
dass beim Hin- und Herfliessen des Wassers oder bei der Bewegung der Luft die in den Poren des Wassers, bezw. der Luft befindlichen Lichttheilchen sich auch bewegen müssten, ohne dass man die Beobachtung davon machen kann, wiewohl die Körper an denen das Licht sich zeigt, in Bewegung sich befinden. (Doch könnte man das Glitzern und Schimmern dem Alex. als Instanz entgegenhalten.)

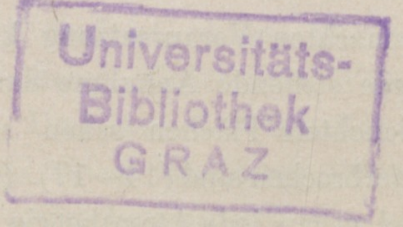

(Sonderabdruck aus dem Archiv für Geschichte der Philosophie IX. Band 2. Heft. 1896.)

Druck von Georg Reimer in Berlin. 